International Journal of Bifurcation and Chaos

(c) World Scientific Publishing Company

\title{
NEW APPROACH TO MODELING SYMBIOSIS IN BIOLOGICAL AND SOCIAL SYSTEMS
}

\author{
V.I. YUKALOV \\ Department of Management, Technology and Economics, \\ ETH Zürich, Swiss Federal Institute of Technology, Zürich CH-8092, Switzerland \\ and \\ Bogolubov Laboratory of Theoretical Physics, \\ Joint Institute for Nuclear Research, Dubna 141980, Russia \\ yukalov@theor.jinr.ru \\ E.P. YUKALOVA \\ Department of Management, Technology and Economics, \\ ETH Zürich, Swiss Federal Institute of Technology, Zürich CH-8092, Switzerland \\ and \\ Laboratory of Information Technologies, \\ Joint Institute for Nuclear Research, Dubna 141980, Russia \\ lyukalov@ethz.ch \\ D. SORNETTE \\ Department of Management, Technology and Economics, \\ ETH Zürich, Swiss Federal Institute of Technology, Zürich CH-8092, Switzerland \\ and \\ Swiss Finance Institute, c/o University of Geneva, \\ 40 blvd. Du Pont d'Arve, CH 1211 Geneva 4, Switzerland \\ dsornette@ethz.ch
}

Received (to be inserted by publisher)

We suggest a novel approach to treating symbiotic relations between biological species or social entities. The main idea is the characterisation of symbiotic relations of coexisting species through their mutual influence on their respective carrying capacities, taking into account that this influence can be quite strong and requires a nonlinear functional framework. We distinguish three variants of mutual influence, representing the main types of relations between species: (i) passive symbiosis, when the mutual carrying capacities are influenced by other species without their direct interactions; (ii) active symbiosis, when the carrying capacities are transformed by interacting species; and (iii) mixed symbiosis, when the carrying capacity of one species is influenced by direct interactions, while that of the other species is not. The approach allows us to describe all kinds of symbiosis, mutualism, commensalism, and parasitism within a unified scheme. The case of two symbiotic species is analysed in detail, demonstrating several dynamical regimes of coexistence, unbounded growth of both populations, growth of one and elimination 
of the other population, convergence to evolutionary stable states, and everlasting population oscillations. The change of the dynamical regimes occurs by varying the system parameters characterising the destruction or creation of the mutual carrying capacities. The regime changes are associated with several dynamical system bifurcations.

Keywords: Mathematical models of symbiosis, nonlinear differential equations, dynamics of coexisting species, functional carrying capacity, dynamical system bifurcations, supercritical Hopf bifurcation 


\section{Introduction}

Symbiosis is one of the most widespread phenomenon in nature. Actually, practically all existing species enjoy some kind of symbiotic relations. As examples, we can start from ourselves, as human beings, whose bodies are in symbiotic relations with ten thousand or more bacterial species (National Institute of Health, USA, http://www.nih.gov/news/health/jun2012/nhgri-13.htm) and around $10^{14}$ different microorganisms. Other well known examples include the association between plant roots and fungi, between coral organisms and various types of algae, between cattle egrets and cattle, between alive beings and different bacteria and viruses. Numerous other examples can be found in the literature Boucher, 1988; Douglas, 1994; Sapp, 1994; Ahmadjian \& Paracer, 2000; Townsend et al., 2002; Yukalov et al., 2012a].

Symbiotic relations are not restricted only to biological species, but may be noticed in a variety of economic, financial, and other relations in social systems. For example, as a variant of symbiosis one can treat the interconnections between economics and arts, between basic and applied science, between culture and language, between banks and firms, between different enterprises, between firm owners and hired personal, and so on. Many more examples are discussed in the following publications Von Hippel, 1988; Grossman \& Helpman, 1991; Richard, 1993; Graedel \& Allenby, 2003; Yukalov et al., 2012a].

Coexistence of different species, such as predators and preys, is usually described by the evolution equations of the Lotka-Volterra type [Lotka, 1925], where the species directly interact with each other. Such equations are not valid for describing symbiosis since, in symbiotic relations, the species do not eat or kill each other, but influence their mutual livelihoods Boucher, 1988; Douglas, 1994; Sapp, 1994; Ahmadjian \& Paracer, 2000; Townsend et al., 2002; Yukalov et al., 2012a, b].

From the mathematical point of view, the replicator equations characterizing trait groups, e.g., cooperators, defectors, and punishers, are also of the Lotka-Volterra type, as they consider direct interactions. This also concerns the public-goods games for the spatially structured trait-group coexistence on networks Perc \& Szolnoki, 2010, 2012; Szolnoki \& Perc, 2013a, bi; Perc et al., 2013], as well as the utility-rate equations [Yukalov et al., 2013]. Such equations do not seem to be applicable for describing symbiotic relations.

The special feature of symbiotic relations is that the coexisting species interact with each other mainly through changing the carrying capacities of each other Boucher, 1988; Douglas, 1994; Sapp, 1994; Ahmadjian \& Paracer, 2000; Townsend et al., 2002; Yukalov et al., 2012a]. Therefore, for the correct description of symbiosis, it is necessary to consider the species carrying capacities as functionals of the species population fractions. In the previous publications [Yukalov et al., 2012a, $\mathrm{b}$ ], the species carrying capacity functionals were considered as polynomial expansions in powers of population fractions, with taking into account only the lowest orders of these expansions, which resulted in linear or bilinear approximations, depending on the symbiosis type. Such lowest-order approximations presuppose that the symbiotic interactions are sufficiently weak, only slightly varying the mutual carrying capacities. The formal use of the linear or bilinear approximations in some cases leads to artificial finite-time singularity or finite-time population death. This defect could be avoided by employing a nonlinear form for the carrying capacities.

It is the aim of the present paper to formulate the evolution equations for coexisting symbiotic species interacting through the influence on the carrying capacities of each other, keeping in mind that this influence can be arbitrarily strong. This implies the use of a nonlinear form for the carrying capacity functional. We follow the idea used for the generalization of the evolution equation for a single population Yukalov et al., 2014], where some artificial singularities could be eliminated by a nonlinear carrying-capacity functional. But now, we consider several symbiotic coexisting species, which makes the principal difference from the single-species evolution.

In Sec. 2, we formulate the main idea of the approach for describing symbiosis, not as a direct interaction of different species as in the Lotka-Volterra equation but, through the mutual influence on the carrying capacities of each other. We distinguish three types of the mutual influence characterizing passive symbiosis, active symbiosis, and mixed-type symbiosis. The origin of the problems spoiling the linear or bilinear approximations is explained. In Sec. 3, we introduce the generalized formulation for the symbiotic equations with nonlinear carrying-capacity functionals. We stress that this formulation allows one to treat symbiotic relations of arbitrary strength and of any type, such as mutualism, commensalism, and parasitism. In the following sections, we give a detailed analysis of the dynamics and of the evolutionary stable states for 
each kind of the symbiotic relations characterizing passive symbiosis (Sec. 4), active symbiosis (Sec. 5), and mixed symbiosis (Sec. 6) for the cases of mutualism and parasitism. All possible dynamical regimes are investigated, occurring as a result of bifurcations. Of special interest is a supercritical Hopf bifurcation happening in the case of mixed symbiosis. Section 7 summarizes the results for commensalism that is a marginal case between mutualism and parasitism. Section 8 concludes.

\section{Modeling Symbiosis by Mutual Influence on Carrying Capacities}

\subsection{General structure of equations}

Suppose several species, enumerated as $i=1,2, \ldots, S$, coexist, being in mutual symbiosis with each other. It is always admissible to reduce the consideration to dimensionless units, as has been thoroughly explained in the previous articles [Yukalov et al., 2012a, b]. So, in what follows, we shall deal with dimensionless quantities, such as the species fractions $x_{i}=x_{i}(t)$ depending on dimensionless time $t \geq 0$. The species can proliferate, with the inverse of the proliferation rates characterizing their typical lifetimes. Generally, in applications of dynamical theory, the time scales of the connected equations could be different Desroches et al., 2012]. However, in the case of symbiosis, one has to keep in mind that the relations between the species, by definition, are assumed to last sufficiently long to be treated as genuinely symbiotic Boucher, 1988; Douglas, 1994; Sapp, 1994; Ahmadiian \& Paracer, 2000; Townsend et al., 2002; Yukalov et al., 2012a]. While this does not cover all symbiotic relationships, such as those between longlived humans and very short-lived bacterias for instance, we will restrict our considerations to the class of symbiotic relationships in which the lifetimes of the involved species are comparable. This corresponds to the situations where these species compete for the corresponding carrying capacities $y_{i}$. Since the meaning of symbiotic relations is the variation of the mutual carrying capacities, the latter have to be functionals of the population fractions:

$$
y_{i}=y_{i}\left(x_{1}, x_{2}, \ldots, x_{S}\right) .
$$

We consider the society, composed of the symbiotic species, as being closed, so that the carrying capacities are not subject to variations caused by external forces, but are only influenced by the mutual interactions of the coexisting species. Therefore the population dynamics is completely defined by the type of symbiotic relations between the species. In the other case, when external forces would be present, the society dynamics would also be governed by such external forces and would strongly depend on the kind of chosen forces Leonov \& Kuznetsov, 2007; Pongvuthithum \& Likasiri, 2010].

The evolution equation for an $i$-th species takes the form

$$
\frac{d x_{i}}{d t}=x_{i}-\frac{x_{i}^{2}}{y_{i}}
$$

where $x_{i}$ and $y_{i}$ are non-negative. In particular, for the case of two species, $x_{1} \equiv x$ and $x_{2} \equiv z$, we have

$$
\frac{d x(t)}{d t}=x(t)-\frac{x^{2}(t)}{y_{1}(x, z)}, \quad \frac{d z(t)}{d t}=z(t)-\frac{z^{2}(t)}{y_{2}(x, z)} .
$$

To proceed further, it is necessary to specify the expressions for $y_{i}$. It is possible to distinguish three types of symbiosis, depending on the process controlling the variations of the mutual carrying capacities.

\subsection{Passive weak symbiosis}

To clearly characterize the difference between the symbiosis types, we start with the case of weak symbiosis, when the mutual influence on the carrying capacities is not strong, so that the carrying-capacity functionals can be approximated by power-law expansions over the species fractions, limiting ourselves by the lowest orders of such expansions. In what follows, we consider the case of two symbiotic species.

Probably the most often met type of symbiosis is when the species influence the mutual carrying capacities just by the existence of the species themselves, resulting in the change on the mutual livelihoods caused by the species vital activity. For instance, most land ecosystems rely on symbiosis between the plants 
that extract carbon from the air and mycorrhizal fungi extracting minerals from the ground. If the mutual influence is weak, the corresponding carrying capacities can be modeled by the linear approximation

$$
y_{1}(x, z) \simeq 1+b_{1} z, \quad y_{2}(x, z) \simeq 1+g_{1} x,
$$

in which $b_{1}$ and $g_{1}$ are the parameters characterizing the productive or destructive influence of the related species on their counterparts. This type of symbiosis can be termed passive, since the species do not directly interact in the process of varying the livelihoods of their neighbors, in the sense that the impacts of species abundance on carrying capacities are linear.

\subsection{Active weak symbiosis}

If in the process of influencing the livelihoods of each other the species directly interact, then their carrying capacities are approximated by the bilinear expressions

$$
y_{1}(x, z) \simeq 1+b_{2} x z, \quad y_{2}(x, z) \simeq 1+g_{2} x z,
$$

assuming that their interactions are sufficiently weak. The parameters $b_{2}$ and $g_{2}$ again can be interpreted as production or destruction coefficients, according to the production or destruction affecting the related carrying capacities. Examples of this type of symbiosis could be the relations between different firms producing goods in close collaboration with each other, or the relation between basic and applied sciences.

\subsection{Mixed weak symbiosis}

The intermediate type of symbiosis is when one of the species, in the process of influencing the carrying capacity, interacts with the other species, while the latter acts on the carrying capacity of the neighbor just by means of its vital activity, without explicit interactions. This type of symbiosis is common for many biological and social systems, when one of them is a subsystem of the larger one. Thus, such relations exist between the country gross domestic product and the level of science, or between culture and language. In the case of weak influence of this type, the carrying capacities are represented as the expansions

$$
y_{1}(x, z) \simeq 1+b_{2} x z, \quad y_{2}(x, z) \simeq 1+g_{1} x .
$$

\section{Arbitrarily Strong Influence on Livelihoods of Symbiotic Species}

When one or both of the species are parasites, the corresponding parameters $b_{i}$ or $g_{i}$ are negative. Hence the effective carrying capacity $y_{i}$ can become zero or negative. Then, with the approximations (4), (5) or (6), the evolution equations (3) can result in finite-time singularities of the population fraction or in the disappearance of the solutions, as is found in the previous publications [Yukalov et al., 2012a, b]. Such a situation looks artificial, being just the result of the linear or bilinear approximations involved. In order to avoid that carrying capacities can become zero and negative, it is necessary to employ a more elaborated expression for the carrying-capacity functionals.

\subsection{Passive nonlinear symbiosis}

In order to be able to consider mutual symbiotic relations of arbitrary strength, it is necessary to generalize the carrying capacities. The latter can be treated as expansions in powers of the species fractions. In the case of weak symbiosis, it has been possible to limit oneself to the first terms of such expansions. However, for generalizing the applicability of the approach, it is necessary to define the effective sums of such expansions. The effective summation of power series can be done by means of the self-similar approximation theory Yukalov, 1990, 1991, 1992; Yukalov \& Yukalova, 1996; Gluzman \& Yukalov, 1997]. Under the condition of obtaining an effective sum that does not change its sign for the variables defined on the whole real axis, one needs to resort to the exponential approximants Yukalov \& Gluzman, 1998; Gluzman \& Yukalov, 1998a, b. c; Yukalov \& Gluzman, 1999; Gluzman et al., 2003]. Limiting ourselves by the simplest form of such an exponential approximation, for the case of expansion (4), we have the effective sums

$$
y_{1}(x, z)=e^{b z}, \quad y_{2}(x, z)=e^{g x} .
$$


The parameters $b$ and $g$, depending on their signs, characterize the creative or destructive influence of the species on the carrying capacities of their coexisting neighbors.

\subsection{Active nonlinear symbiosis}

Similarly involving the method of self-similar exponential approximants to perform the summation for the previous case of passive symbiosis, starting from the terms (5), we obtain the nonlinear carrying capacities

$$
y_{1}(x, z)=e^{b x z}, \quad y_{2}(x, z)=e^{g x z},
$$

with the same interpretation of the symbiotic parameters $b$ and $g$.

\subsection{Mixed nonlinear symbiosis}

In the case of the mixed symbiosis, with the carrying capacities starting from terms (6), we get the effective summation resulting in the nonlinear expressions

$$
y_{1}(x, z)=e^{b x z}, \quad y_{2}(x, z)=e^{g x} .
$$

In this way, we obtain the generalizations for the carrying capacities that are applicable to the mutual symbiotic relations of arbitrary strength.

\subsection{Variants of symbiotic coexistence}

It is worth stressing that the suggested approach allows us to treat all known variants of symbiosis, which is regulated by the values of the symbiotic parameters $b$ and $g$. Thus, when both these parameters are positive, this corresponds to mutualism, which is a relationship between different species where both of them derive mutual benefit:

$$
b>0, \quad g>0 \quad(\text { mutualism }) .
$$

When one of the species benefits from the coexistence with the other species, while the other one is neutral, getting neither profit nor harm, this relationship corresponds to commensalism, defined by one of the conditions

$$
\begin{array}{lll}
b>0, & g=0 ; \\
b=0, & g>0
\end{array} \quad \text { (commensalism). }
$$

Finally, if at least one of the coexisting species is harmful to the other one, this is typical of parasitism that is characterized by the validity of one of the conditions

$$
\begin{array}{ll}
b>0, & g<0 ; \\
b=0, & g<0 ; \\
b<0, & g<0 ; \\
b<0, & g=0 ; \\
b<0, & g>0 \quad \text { (parasitism). }
\end{array}
$$

Varying the symbiotic parameters $b$ and $g$ results in a variety of bifurcations between different dynamical regimes. Below, we study these bifurcations employing the general methods of dynamical theory Thompson et al., 1994; Kuznetsov, 1995; Chen et al., 2003; Leonov \& Kuznetsov, 2013].

\section{Passive Nonlinear Symbiosis}

Equations (3), with the carrying capacities (7) acquire the form

$$
\frac{d x}{d t}=x-x^{2} e^{-b z}, \quad \frac{d z}{d t}=z-z^{2} e^{-g x}
$$

with the parameters $g \in(-\infty, \infty)$ and $b \in(-\infty, \infty)$, and with the initial conditions $x_{0}=x(0), z_{0}=z(0)$. We are looking for non-negative solutions $x(t) \geq 0$ and $z(t) \geq 0$ to the system of these differential equations (13). 
We may note that system (13) is symmetric with respect to the change $b \longleftrightarrow g, x_{0} \longleftrightarrow z_{0}$, and $x(t) \longleftrightarrow z(t)$

The overall phase portrait for the case of the passive symbiosis will be displayed below.

\subsection{Existence of evolutionary stable states}

Depending on the signs of the parameters $g$ and $b$, the system of equations (13) possesses different numbers of stationary states (fixed points) $\left\{x^{*}, z^{*}\right\}$. For any values of the parameters, there always exist three trivial fixed points, $\left\{x^{*}=0, z^{*}=0\right\},\left\{x^{*}=1, z^{*}=0\right\}$, and $\left\{x^{*}=0, z^{*}=1\right\}$, which are unstable for all $g$ and $b$.

Nontrivial fixed points $\left\{x^{*} \neq 0, z^{*} \neq 0\right\}$ are the solutions to the equations:

$$
x^{*}=e^{b z^{*}}, \quad z^{*}=e^{g x^{*}} .
$$

The characteristic exponents, defining the stability of the stationary solutions, are given by the expression

$$
\lambda_{1,2}=-1 \pm \sqrt{b g x^{*} z^{*}},
$$

where $x^{*}$ and $z^{*}$ are the fixed points defined by Eqs. (14), which can also be represented as

$$
x^{*}=\exp \left(b e^{g x^{*}}\right), \quad z^{*}=\exp \left(g e^{b z^{*}}\right) .
$$

Analysing the existence and stability of the fixed points, we find that, when $b \in(0, \infty)$ and $g \in(0, \infty)$, there exists a line $g=g_{c}(b)$, with respect to which two possibilities can occur:

- If $0<g<g_{c}(b)$, then Eqs. (14) have two solutions $\left\{x_{1}^{*}, z_{1}^{*}\right\}$ and $\left\{x_{2}^{*}, z_{2}^{*}\right\}$, such that $1<x_{1}^{*}<x_{2}^{*}$ and $1<z_{1}^{*}<z_{2}^{*}$. Numerical investigation of (14) and (15) shows that the lower fixed point $\left\{x_{1}^{*}, z_{1}^{*}\right\}$ is stable, while the higher fixed point $\left\{x_{2}^{*}, z_{2}^{*}\right\}$ is unstable.

- If $g>g_{c}(b)$, then Eqs. (14) do not have solutions, hence Eqs. (13) do not possess stationary states. When $b=1 / e$, then $g_{c}(b)=1 / e$ and $x^{*}=z^{*}=e$.

If either $b \leq 0$ and $g>0$, or $b>0$ and $g \leq 0$, then there exists only one stationary solution $\left\{x^{*}, z^{*}\right\}$, which is a stable fixed point characterized by the corresponding characteistic exponents (15) with $\operatorname{Re} \lambda_{1,2}<0$, which follows from the inequalities $\lambda_{1} \lambda_{2}=1-b g x^{*} z^{*}>0$ and $-\left(\lambda_{1}+\lambda_{2}\right)=2>0$.

Note that if $b \leq 0$ and $g>0$, then $x^{*}<1$ and $z^{*}>1$, while if $b>0$ and $g \leq 0$, then $x^{*}>1$ and $z^{*}<1$.

When $b<0$ and $g<0$, there can exist up to three fixed points, such that $x^{*}<1$ and $z^{*}<1$. For $b \leq-e$, there exist two lines $g_{1}=g_{1}(b)<0$ and $g_{2}=g_{2}(b)<0$, for which $g_{1}(-e)=g_{2}(-e)=-e$. The following two possibilities can happen:

- If $b<-e$ and $g_{1}(b)<g<g_{2}(b)$, then Eqs. (14) have 3 solutions. Numerical analysis shows that two fixed points, $\left\{x_{1}^{*}, z_{1}^{*}\right\}$ and $\left\{x_{2}^{*}, z_{2}^{*}\right\}$, are stable, while the third fixed point, $\left\{x_{3}^{*}, z_{3}^{*}\right\}$, is unstable.

- If either $-e \leq b<0$ and $g<0$, or $b<-e$ and $g<g_{1}(b)$, or $b<-e$ and $g_{2}(b)<g<0$, then Eqs. (14) have only one solution, which is a stable fixed point.

In the limiting cases, if $g \rightarrow 0$, then $z^{*} \rightarrow 1$ and $x^{*} \rightarrow e^{b}$. If $b \rightarrow 0$, then $x^{*} \rightarrow 1$ and $z^{*} \rightarrow e^{g}$.

If $b \rightarrow-\infty$ and $g>0$ is fixed, then $x^{*} \rightarrow 0$ and $z^{*} \rightarrow 1$. When $g \rightarrow-\infty$ and $b>0$ is fixed, then $z^{*} \rightarrow 0$ and $x^{*} \rightarrow 1$.

If $b \ll-1$, and $g \ll-1$, then, depending on the relation between the parameters, it may be that either $x^{*} \rightarrow 0$ and $z^{*} \rightarrow 1$, or $x^{*} \rightarrow 1$ and $z^{*} \rightarrow 0$, or $x^{*} \rightarrow 0$ and $z^{*} \rightarrow 0$, but at finite parameter values, one always has finite $x^{*} \neq 0$ and $z^{*} \neq 0$.

Figure 1a clarifies the regions of the stable fixed-point existence in the plane $b-g$. The region of existence for $b, g<0$ is detailed in Fig. $1 \mathrm{~b}$.

\subsection{Dynamics of populations under mutualism $(b>0, g>0)$}

There exist two types of dynamical behavior: (i) Unbounded growth of populations or (ii) convergence to a stationary state. 
(i) Unbounded growth of populations

When $0<b<\infty$ and $g>g_{c}(b)>0$, then Eqs. (14) do not have solutions, hence Eqs. (13) do not have stationary states. For any choice of the initial conditions $\left\{x_{0}, z_{0}\right\}$, solutions $x(t), z(t) \rightarrow \infty$, as $t \rightarrow \infty$. The corresponding behavior of $x(t)$ and $z(t)$ is shown in Fig.2a.

(ii) Convergence to stationary states

If $0<b<\infty$ and $0<g<g_{c}(b)$, there exist two solutions to Eqs. (14), such that $1<x_{1}^{*}<x_{2}^{*}$ and $1<z_{1}^{*}<z_{2}^{*}$. Numerical analysis shows that the lower solution $\left\{x_{1}^{*}, z_{1}^{*}\right\}$ is stable, while $\left\{x_{2}^{*}, z_{2}^{*}\right\}$ is unstable. Around the stable fixed point, there exists a finite basin of attraction, so that if the initial conditions $\left\{x_{0}, z_{0}\right\}$ belong to it, then $x(t) \rightarrow x_{1}^{*}$, and $z(t) \rightarrow z_{1}^{*}$, as $t \rightarrow \infty$. But if the initial conditions do not belong to the basin of attraction, the species fractions rise to infinity for $t \rightarrow \infty$.

Figure $2 \mathrm{~b}$ demonstrates the behavior or solutions for $g$ slightly above the boundary $\left(g>g_{c}(b)\right)$ and slightly below it $\left(g<g_{c}(b)\right)$. Below the boundary $g_{c}=g_{c}(b)$, there exists a region of parameters $b, g$, such that $x(t)$ and $z(t)$ converge to a stationary state, provided that the initial conditions are inside the basin of attraction. Figures $2 \mathrm{c}$ and $2 \mathrm{~d}$ illustrate the behaviour of $x(t)$ and $z(t)$ for the same choice of the parameters $b, g$, but for different initial conditions.

\subsection{Dynamics of populations with one parasitic species (either $b<0, g>0$ or $b>0, g<0)$}

Only one regime exists, when the populations tend to their stationary states, with the attraction basin being the whole region of $x_{0}, z_{0}$.

Recall that there is a symmetry in Eqs. (13), such that under the replacement $b \longleftrightarrow g$ and $x_{0} \longleftrightarrow z_{0}$, we have $x(t) \longleftrightarrow z(t)$ and $x^{*} \longleftrightarrow z^{*}$. Therefore, it is sufficient to consider only one case, say, when $b<0$ and $g>0$.

When $b<0$ and $g>0$, Eqs. (14) have a single solution $\left\{x^{*}<1, z^{*}>1\right\}$, which is a stable fixed point. Conversely, when $b>0$ and $g<0$, then $x^{*}>1$ and $z^{*}<1$. This tells us that the species population coexisting with a parasite is suppressed.

The populations converge to their stationary points irrespectively of the choice of the initial conditions. The convergence can be either monotonic or non-monotonic, as is demonstrated in Figs. 3 and 4.

\subsection{Dynamics of populations with two parasitic species $(b<0, g<0)$}

Depending on the symbiotic parameters, there can exist either (i) a single stationary state or (ii) bistability with two stationary states.

When $b<0$ and $g<0$, then Eqs. (14) have either a single solution $\left\{x^{*}, z^{*}\right\}$, which is a stable fixed point, or three solutions, among which two solutions, $\left\{x_{1,2}^{*}, z_{1,2}^{*}\right\}$, are stable fixed points and the third, $\left\{x_{3}^{*}, z_{3}^{*}\right\}$, is a saddle. In all the cases, $x^{*}<1$ and $z^{*}<1$, which means that two parasitic species cannot develop large populations.

(i) Single stationary state

For $b, g \leq-e$, there exist two lines, $g_{1}(b)$ and $g_{2}(b)$, such that $g_{1}(-e)=g_{2}(-e)=-e$ and $g_{1}(b)<$ $g_{2}(b)<0$. If either $-e<b<0$ and $g<0$, or $b<-e$ and $g_{2}(b)<g<0$, or $b<-e$ and $g<g_{1}(b)$, then there exists a single solution to Eqs. (14), which is a stable fixed point.

(ii) Bistability with two stationary states.

If $b<-e$ and $g_{1}(b)<g<g_{2}(b)$, there exist three solutions to Eqs. (14). Two solutions are stable fixed points and the third solution is a saddle. The bifurcation point corresponds to $b=g=-e$. At this point, $x^{*}=z^{*}=1 / e$.

The populations $x(t)$ and $z(t)$ tend monotonically or non-monotonically to their stable stationary states, from above or from below, as is shown in Figs. 5 and 6 . In the region of the symbiotic parameters $b, g<0$, where a single stable fixed point exists, $x(t) \rightarrow x^{*}, z(t) \rightarrow z^{*}$, as $t \rightarrow \infty$, irrespectively of the initial conditions. 
In the region of the parameters $b$ and $g$, where two stable fixed point exist, the population convergence depends on the choice of initial conditions. For $t \rightarrow \infty$, the population $\{x(t), z(t)\}$ tend either to $\left\{x_{1}^{*}, z_{1}^{*}\right\}$ or to $\left\{x_{2}^{*}, z_{2}^{*}\right\}$, depending on the initial conditions $\left\{x_{0}, z_{0}\right\}$ being in the attraction basin of the related fixed point.

On the line $-e<b=g<0$, there exists a single stationary state $\left\{x^{*}=z^{*}\right\}$ that is a stable fixed point.

On the line $b=g<-e$, there are three fixed points. Two fixed points are stable, so that $x_{1}^{*}=z_{2}^{*}$ and $z_{1}^{*}=x_{2}^{*}$. The third fixed point, $\left\{x_{3}^{*}=z_{3}^{*}\right\}$ is unstable. For $b=g \rightarrow-\infty$, we have $x_{1}^{*} \rightarrow 0, z_{1}^{*} \rightarrow 1, x_{2}^{*} \rightarrow 1$, $z_{2}^{*} \rightarrow 0$, and $x_{3}^{*}=z_{3}^{*} \rightarrow 0$.

\subsection{Phase portrait for passive nonlinear symbiosis}

Fig. 7 provides an overview of the different regimes analysed in this section concerned with the analysis of equation (13).

Panels (a) and (b) illustrate the regime of mutualism, in which the two species benefit from each other. Panel (a) represents the situation of sufficiently symmetric mutualism, in which the long-term behaviour is characterised by an exponential growth for both species. Note that asymmetric initial conditions lead to a non-monotonous behaviour of the species population that is initially too large, which has to shrink first before growing again in synergy with the other species. Panel (b) corresponds to the situation of large mutualism asymmetries. In this case, the two population need to be above a certain threshold in order to reach the regime of unbounded growth. Otherwise, their populations are trapped and converge to a steady state.

Panel (c) illustrates the dynamics of a very asymmetric situation where one species augments the carrying capacity of the other, while the later has a negative effect on the carrying capacity of the former. In this case, the dynamics of the two species population converges to a stable fixed point, a steady state characterising a compromise in the destructive interactions between the two species.

In the situation shown in panel (d) in which both species tend to destroy the carrying capacity of the other, a saddle node separates two symmetrical stable fixed points, where one species in general profits much more than the other. This is an example of a spontaneous symmetry breaking, in which the two stable fixed points are exactly symmetric under the transformation $x \longleftrightarrow z$. The selection of one or the other stable fixed points depends on the initial conditions, as usual in spontaneous symmetry breaking. A slight initial advantage of $x$ above $z$ is sufficient to consolidate into a very large asymptotic population difference. Such behaviours are reminiscent to many real-life situations in which a slight initial favourable situation becomes entrenched in a very strong prominent role. Our analysis shows that such a situation occurs generically in the case of passive symbiosis when the two species are competing destructively. It is tempting to interpret real-life geopolitical and economic situations in particular as embodiment of such a scenario.

\section{Active Nonlinear Symbiosis}

This case is described by the system of equations

$$
\frac{d x}{d t}=x-x^{2} e^{-b x z}, \quad \frac{d z}{d t}=z-z^{2} e^{-g x z},
$$

with the symbiotic parameters $g \in(-\infty, \infty), b \in(-\infty, \infty)$, and the initial conditions $x_{0}=x(0), z_{0}=z(0)$. Again, only non-negative solutions $x(t) \geq 0$ and $z(t) \geq 0$ are of interest. Note that the system (16) is symmetric with respect to the replacement $b \longleftrightarrow g, x_{0} \longleftrightarrow z_{0}$, and $x(t) \longleftrightarrow z(t)$.

\subsection{Existence of evolutionary stable states}

Similarly to the previous case, there always exist three trivial fixed points, $\left\{x^{*}=0, z^{*}=0\right\},\left\{x^{*}=1, z^{*}=\right.$ $0\}$, and $\left\{x^{*}=0, z^{*}=1\right\}$, which are unstable for any $g$ and $b$.

Nontrivial fixed points $\left\{x^{*} \neq 0, z^{*} \neq 0\right\}$ are the solutions to the equations:

$$
x^{*}=e^{b x^{*} z^{*}}, \quad z^{*}=e^{g x^{*} z^{*}} .
$$


These Eqs. (17) can be represented as

$$
x^{*}=\exp \left(b\left(x^{*}\right)^{1+g / b}\right), \quad z^{*}=\exp \left(g\left(z^{*}\right)^{1+b / g}\right) .
$$

The characteristic exponents are given by the equations

$$
\lambda_{1}=-1, \quad \lambda_{2}=-1+(b+g) x^{*} z^{*},
$$

where $x^{*}$ and $z^{*}$ are the solutions to Eqs. (17). From here, it follows that the fixed points are stable for all $b+g<0$.

The following situations can occur:

- If $b+g>1 / e$, then Eqs. (17), or (18), do not have solutions.

- If $0<b+g<1 / e$, then Eqs. (17) or (18) have two solutions $\left\{x_{1}^{*}, z_{1}^{*}\right\}$ and $\left\{x_{2}^{*}, z_{2}^{*}\right\}$.

Here, when $b>0, g>0$, then $1<x_{1}^{*}<x_{2}^{*}$ and $1<z_{1}^{*}<z_{2}^{*}$. When $b<0$ and $g>0$, then $1>x_{1}^{*}>x_{2}^{*}$ and $1<z_{1}^{*}<z_{2}^{*}$. If $b>0$ and $g<0$, then $1<x_{1}^{*}<x_{2}^{*}$, but $1>z_{1}^{*}>z_{2}^{*}$. Numerical investigation shows that the fixed point $\left\{x_{1}^{*}, z_{1}^{*}\right\}$ is stable, while the second fixed point $\left\{x_{2}^{*}, z_{2}^{*}\right\}$ is a saddle.

- If $b+g<0$, then there exists only one solution to Eqs. (17) or (18) that is a stable fixed point.

The region of the existence of the stable fixed-point in the plane $b-g$ is presented in Fig. 8 .

When $0<b+g<1 / e$, the stable fixed point possesses a finite basin of attraction. If $b+g \leq 0$, then the basin of attraction is the whole region $\left\{x_{0} \geq 0, z_{0} \geq 0\right\}$.

\subsection{Population dynamics under $b+g>1 / e$}

In this case, Eqs. (17) or (18) do not have solutions, which means that there are no fixed points. There can happen two types of solutions: (i) Unbounded growth of mutualistic populations or (ii) Unbounded growth of a parasitic population and dying out of the host population.

(i) Unbounded growth of mutualistic populations

In the case of mutualism, when $b>0$ and $g>0$, both populations grow so that $x(t) \rightarrow \infty$ and $z(t) \rightarrow \infty$, as $t \rightarrow \infty$.

(ii) Unbounded growth of parasitic population and dying out of host population

When one of the species is parasitic, the host population dies out. If $b<0$, but $g>0$, then $x(t) \rightarrow 0$ and $z(t) \rightarrow \infty$, as $t \rightarrow \infty$. Conversely, if $b>0$, but $g<0$, then $x(t) \rightarrow \infty$, while $z(t) \rightarrow 0$, as $t \rightarrow \infty$.

The population dynamics for the case $b+g>1 / e$ is presented in Fig. 9. Because of the symmetry of Eqs. (16), it is sufficient to consider only the cases when $b>0, g>0$ and $b<0, g>0$.

\subsection{Population dynamics under $b+g<1 / e$}

There exists only one stable fixed point, so that the population dynamics depends on whether the initial conditions are inside the attraction basin or not. Respectively, there can happen three kinds of behavior: (i) Convergence to a stationary state; (ii) Unbounded growth of both populations; (iii) Unbounded growth of parasitic population and dying out of host population.

(i) Convergence to stationary state

When $0<b+g<1 / e$ and the initial conditions $\left\{x_{0}, z_{0}\right\}$ are in the basin of attraction of the fixed point $\left\{x^{*}, z^{*}\right\}$, then the populations $x(t) \rightarrow x^{*}$ and $z(t) \rightarrow z^{*}$, as $t \rightarrow \infty$.

If $b+g \leq 0$, then the basin of attraction is the whole region of $x_{0} \geq 0$ and $z_{0} \geq 0$, that is, for any choice of the initial conditions $\left\{x_{0}, z_{0}\right\}$ the solutions $\{x(t), z(t)\} \rightarrow\left\{x^{*}, z^{*}\right\}$, as $t \rightarrow \infty$.

(ii) Unbounded population growth

When $0<b+g<1 / e$ and $b, g>0$, there exists a finite basin of attraction, so that if the initial conditions $\left\{x_{0}, z_{0}\right\}$ are outside of the attraction basin, then the populations $\{x(t), z(t)\}$ unboundedly grow with increasing time $t \rightarrow \infty$. 
(iii) Unbounded growth of parasitic population and dying out of host population

When $0<b+g<1 / e$, with $b<0$ but $g>0$, and initial conditions $\left\{x_{0}, z_{0}\right\}$ are outside of the attraction basin, then for $t \rightarrow \infty$, one of the species is dying out, $x(t) \rightarrow 0$, while another one experiences unbounded growth $z(t) \rightarrow \infty$. Conversely, if $b>0$, but $g<0$, then $x(t) \rightarrow \infty$, while $z(t) \rightarrow 0$, as $t \rightarrow \infty$.

Figure 10 demonstrates different types of the population convergence to a stable stationary state $\left\{x^{*}, z^{*}\right\}$.

\subsection{Phase portrait for active nonlinear symbiosis}

Figure 11 provides an overview of the different regimes analysed in this section concerned with the analysis of equation (16). Qualitatively, the nature of the different regimes are similar to those discussed with respect to the phase portrait shown in Fig. 7 summarising the different regime for passive symbiosis described by equation (13).

\section{Mixed Nonlinear Symbiosis}

The system of equations describing this case is

$$
\frac{d x}{d t}=x-x^{2} e^{-b x z}, \quad \frac{d z}{d t}=z-z^{2} e^{-g x},
$$

with the symbiotic parameters $g \in(-\infty, \infty), b \in(-\infty, \infty)$, and the initial conditions $x_{0}=x(0), z_{0}=z(0)$. As usual, only non-negative solutions $x(t) \geq 0$ and $z(t) \geq 0$ are of interest.

Note that contrary to Eqs. (13) and (16), the system of equations (20) is not symmetric. The carrying capacity of the population $x(t)$ is formed in the process of interactions between the coexisting species. In contrast, the carrying capacity of the population $z(t)$ is influenced only by the population $x(t)$. Therefore, we may call the population $x(t)$ active, but the population $z(t)$ passive.

\subsection{Existence of evolutionary stable states}

Similarly to the previous cases, there always exist 3 trivial fixed points, $\left\{x^{*}=0, z^{*}=0\right\},\left\{x^{*}=1, z^{*}=0\right\}$, and $\left\{x^{*}=0, z^{*}=1\right\}$, which are unstable for all $b, g \in(-\infty, \infty)$.

Nontrivial fixed points $\left\{x^{*} \neq 0, z^{*} \neq 0\right\}$ are the solutions to the equations:

$$
x^{*}=e^{b x^{*} z^{*}}, \quad z^{*}=e^{g x^{*}}
$$

which can be transformed into

$$
x^{*}=\exp \left(b x^{*} e^{g x^{*}}\right), \quad z^{*}=\exp \left(g z^{b z^{*} / g}\right) .
$$

The characteristic exponents, defining the stability of the stationary states, are given by the equations

$$
\lambda_{1,2}=\frac{1}{2}\left[b x^{*} z^{*}-2 \pm x^{*} \sqrt{b z^{*}\left(4 g+b z^{*}\right)}\right],
$$

The following cases can occur:

- When $b>0$, there exists a boundary $g=g_{c}(b)$, such that if $g<g_{c}(b)$, then there can exist up to three fixed points, but only one of them being stable.

- If either $0<b<1 / e$ and $g>g_{c}(b)>0$, or $b>1 / e$ and $g \geq 0$, then Eqs. (21) do not have solutions, hence, there are no fixed points.

- For $b>1 / e$ and $g_{c}(b)<g<0$, there exists only one unstable fixed point.

- If $0<b<1 / e$ and $0 \leq g<g_{c}(b)$, there is one stable and one unstable fixed points.

- When $b \leq 0$ and $g \in(-\infty, \infty)$, there exists a single fixed point that is stable. For $b<0$ and $g>0$, we have $x^{*}<1, z^{*}>1$, while when $b<0$ and $g<0$, then $x^{*}<1, z^{*}<1$.

- For $0 \leq b \leq b_{0} \approx 0.47$, there exists an additional line $g_{0}(b)$, such that $g_{0}(0)=0$ and $g_{0}\left(b_{0}\right)=g_{c}\left(b_{0}\right) \approx$ -0.075706 . If either $0<b<b_{0}$ and $g<g_{0}(b)$ or $b \geq b_{0}$ and $g<g_{c}(b)$, there is a single fixed point that is stable. 
- When either $0<b<1 / e$ and $g_{0}(b)<g<0$, or $1 / e \leq b<b_{0}$ and $g_{0}(b)<g<g_{c}(b)$, then Eqs. (21) have three solutions, but only one of them is a stable fixed point.

In the limiting case, we have $g_{c}(1 / e)=0$. When $b \rightarrow+0$, then $g_{c}(b) \rightarrow \infty$. If $b \rightarrow \infty$, then $g_{c}(b) \rightarrow-\infty$. On the boundary $\left\{b \in\left(0, b_{0}\right), g=g_{0}(b)\right\}$, two solutions, corresponding to unstable fixed points, coincide and disappear as soon as $g \leq g_{0}(b)$.

The region of the existence of the fixed-point in the plane $b-g$ is presented in Fig. 12.

The dynamics of the symbiotic populations strongly depends on whether the influence of the passive species $z(t)$ on the active species $x(t)$ is mutualistic or parasitic, which is described by their interaction symbiotic parameter $b$. Therefore, these two cases will be treated separately.

\subsection{Dynamics of populations with mutualistic passive species $(b>0)$}

Depending on the symbiotic parameters $b$ and $g$, there are three types of dynamic behavior: (i) Unbounded growth of populations; (ii) everlasting oscillations, and (iii) convergence to a stationary state.

(i) Unbounded growth of populations

When either $0<b<1 / e$ and $g>g_{c}(b)>0$, or $b>1 / e$ and $g \geq 0$, then Eqs. (20) do not have fixed points. Populations $x(t) \rightarrow \infty$ and $z(t) \rightarrow \infty$, either monotonically or non-monotonically, as $t \rightarrow \infty$. The logarithmic behaviour of diverging solutions $x(t)$ and $z(t)$ is shown in Figs. 13a and 13b.

When $0<b<1 / e$ and $0 \leq g<g_{c}(b)$, there are two fixed points, but only one of them is stable. In this case, there exists a finite basin of attraction for the stable fixed point. If the initial conditions $\left\{x_{0}, z_{0}\right\}$ are outside of the attraction basin, the populations $x(t)$ and $z(t)$ diverge, as $t \rightarrow \infty$.

(ii) Everlasting oscillations

When $b>1 / e$ and $g_{c}(b)<g<0$, then Eqs. (21) have only one solution $\left\{x^{*}, z^{*}\right\}$, which is an unstable focus, with complex characteristic exponents, such that $\lambda_{2}=\lambda_{1}^{*}$ and $\operatorname{Re} \lambda_{1,2}>0$. In this region of the parameters $b$ and $g$, there exists a limit cycle, and the populations $x(t)$ and $z(t)$ oscillate without convergence for all $t>0$.

When $g \rightarrow-0$, then the amplitude of oscillations drastically increases, but remains finite. On the half line, $b>1 / e$ and $g=0$, solutions $x(t)$ and $z(t)$ diverge, as $t \rightarrow \infty$.

The dynamics of populations $x(t)$ and $z(t)$ are shown in Figs. 13c,d, 14a,b, 15c,d, and 16c,d. Figure 17 presents the logarithmic behavior of the populations $x(t), z(t)$ for the fixed $b=0.4$ and varying $g$, such that either $g_{c}(b)<g<0$ or $g_{0}(b)<g<g_{c}(b)$.

(iii) Convergence to stationary states

When $b>0$ and $g<g_{c}(b)$, there exists one stable fixed point $\left\{x^{*}, z^{*}\right\}$.

When $0<b<1 / e$ and $0<g<g_{c}(b)$, then there exists a finite basin of attraction for the fixed point. If the initial conditions $\left\{x_{0}, z_{0}\right\}$ are inside the basin of attraction, then $x(t) \rightarrow x^{*}$ and $z(t) \rightarrow z^{*}$, as $t \rightarrow \infty$.

If either $0<b<1 / e$ and $g \leq 0$, or $b>1 / e$ and $g<g_{c}(b)<0$, then the basin of attraction is the whole region of $\left\{x_{0} \geq 0, z_{0} \geq 0\right\}$. In that case, for any initial conditions, $x(t) \rightarrow x^{*}$ and $z(t) \rightarrow z^{*}$, as $t \rightarrow \infty$.

There are two possible ways of convergence to a stationary state, either with oscillations, when the fixed point is a focus, or without oscillations, when the fixed point is a node.

When $b>b_{0}$ and $g<g_{c}(b)$, and $g$ is in the vicinity of $g=g_{c}(b)$, then $x(t) \rightarrow x^{*}$ and $z(t) \rightarrow z^{*}$ with oscillations. The corresponding behaviour is shown in Figs. 14c,d, 15c,d (solid lines), and Figs. 16c,d (dashed lines).

When $0<b<1 / e$ and $0<g<g_{c}(b)$ and the initial conditions $\left\{x_{0}, z_{0}\right\}$ are inside the attraction basin of the fixed point, then $x(t) \rightarrow x^{*}, z(t) \rightarrow z^{*}$ without explicit oscillations, though the convergence can be either monotonic or non-monotonic.

If either $0<b<1 / e$ and $g \leq 0$, or $1 / e<b<b_{0}$ and $g<g_{c}(b)$, or $b>b_{0}$ and $g \ll g_{c}(b)$, then $x(t) \rightarrow x^{*}$ and $z(t) \rightarrow z^{*}$ without oscillations.

Examples of convergence without oscillations are given in Figs. 15 and 16. 
The bifurcation line $g=g_{c}(b)<0$ for $b>b_{0}$ is the line of a supercritical Hopf bifurcation. It separates the regions where the real parts of the complex characteristic exponents have different signs. When $b>b_{0}$ and $g<g_{c}(b)<0$, then the characteristic exponents of the stable fixed point are complex, with the negative Lyapunov exponents $\operatorname{Re} \lambda_{1,2}<0$. When $b>b_{0}$ and $g_{c}(b)<g<0$, then the stable focus transforms into an unstable focus, with positive Lyapunov exponents $\operatorname{Re} \lambda_{1,2}>0$. At the same time, there appears a stable limit cycle. On the bifurcation line, where $b>b_{0}$ and $g=g_{c}(b)$ the Lyapunov exponents of the focus are zero, $\operatorname{Re} \lambda_{1,2}=0$, as it should be under a Hopf bifurcation Kuznetsov, 1995; Chen et al., 2003; Cobiaga \& Reartes, 2013].

A very interesting behaviour is associated with the transition across the line $g_{c}(b)$, when $1 / e<b<b_{0}$. In the region where either $0<b<1 / e$ and $g_{0}<g<0$, or $1 / e<b<b_{0}$ and $g_{0}(b)<g<g_{c}(b)$, there are three fixed points, a stable node, a saddle, and an unstable focus. In the region $b>1 / e$ and $g>g_{c}(b)$, there is one unstable focus and a limit cycle. When approaching the boundary $g_{c}(b)$, moving from one region to the other, the stable node and saddle become close to each other and on the boundary they coincide, while disappearing to the right from this line $g_{c}(b)$. The unstable focus safely moves through the boundary to the region where a limit cycle appears.

\subsection{Dynamics of populations with parasitic passive species $(b<0)$}

There is only one regime of convergence to stationary states.

When $b \leq 0$ and $g \in(-\infty, \infty)$, there exists just one solution to Eqs. (21), or (22), and it is a stable fixed point. The attraction basin is the whole region of the initial conditions $\left\{x_{0}, z_{0}\right\}$, so that, for any initial condition, populations $x(t) \rightarrow x^{*}$ and $z(t) \rightarrow z^{*}$, as $t \rightarrow \infty$.

The corresponding behaviour of the populations is presented in Fig. 16.

\subsection{Phase portrait for mixed nonlinear symbiosis}

The phase portraits for the case of the mixed symbiosis described by equation (20), under different symbiotic parameters, are presented in Figs. 18 and 19.

Fig. 18 for $g>0$ shows three different regimes that are qualitatively similar to those shown for the passive symbiosis cases in panels (a)-(c) of Fig. 7 .

Fig. 19 corresponds to the situation where $b>0$ and $g<0$, so that the species $x$ tends to destroy the carrying capacity of the species $z$, while the latter tends to augment nonlinearly the carrying capacity of the former. As a consequence of the asymmetry of the equations and of this pair of parameters, either convergent oscillations to a stable fixed point or oscillatory solutions occur.

\section{Commensalism as Marginal Type of Symbiosis (either $b=0$, or $g=0$ )}

\subsection{Independent species $(b=0$ and $g=0)$}

This is the extreme case, when the species are actually independent of each other. If $b=0$ and $g=0$, then the systems of the evolution equations turn into two independent logistic equations

$$
\frac{d x}{d t}=x-x^{2}, \quad \frac{d z}{d t}=z-z^{2}
$$

with the initial conditions $x_{0}=x(0)$ and $z_{0}=z(0)$.

Solutions to these equations are known:

$$
x(t)=\frac{x_{0}}{x_{0}-\left(x_{0}-1\right) e^{-t}}, \quad z(t)=\frac{z_{0}}{z_{0}-\left(z_{0}-1\right) e^{-t}} .
$$

That is, $x(t) \rightarrow x^{*}=1$ and $z(t) \rightarrow z^{*}=1$, as $t \rightarrow \infty$. 


\subsection{Commensalism under $b=0, g \neq 0$}

When $b=0$ and $g \neq 0$ in equation (20), this can be treated as a limiting case of either passive or mixed symbiosis. Then the system of equations (13), or (20), turns into

$$
\frac{d x}{d t}=x-x^{2}, \quad \frac{d z}{d t}=z-z^{2} e^{-g x(t)} .
$$

The solution $x(t)$ to the first equation of (26) is given by the first equation in (25). The stable set of fixed points is defined by the expressions

$$
x^{*}=1, \quad z^{*}=e^{g},
$$

which exist for all $g \in(-\infty, \infty)$. Populations $\{x(t), z(t)\} \rightarrow\left\{x^{*}, z^{*}\right\}$ monotonically either from above or from below, depending on the given initial conditions.

\subsection{Commensalism under $b \neq 0, g=0$}

When $b \neq 0$ and $g=0$ in equation (20), this is the limiting case of either active or mixed symbiosis. The system of equations (16), or (20), becomes

$$
\frac{d x}{d t}=x-x^{2} e^{-b x z}, \quad \frac{d z}{d t}=z-z^{2} .
$$

The solution $z(t)$ to the second equation of (27) is given by the second expression in (25). The fixed point $z^{*}=1$ is stable, while the fixed point $z^{*}=0$ is unstable.

For the first equation in (27), the trivial fixed point $x^{*}=0$ is also unstable. Nontrivial fixed points $x^{*} \neq 0$ are the solutions to the equation

$$
x^{*}=e^{b x^{*}} .
$$

When $b \leq 0$ there exists a single fixed point $x^{*} \leq 1$ that is stable. Then, for any initial conditions, $x(t) \rightarrow x^{*}$ and $z(t) \rightarrow z^{*}=1$, as $t \rightarrow \infty$.

If $0<b<1 / e$, there exist two solutions, but only one solution, such that $1<x^{*}<e$, is stable, possessing a finite basin of attraction.

When $b>1 / e$, there are no fixed points for $x(t)$, hence $x(t) \rightarrow \infty$, though $z(t) \rightarrow 1$, as $t \rightarrow \infty$.

Dynamics of the populations $x(t)$ and $z(t)$ in the case of commensalism, described by Eqs. (24), (26), and (27), is demonstrated in Fig. 20.

\section{Summary of Main Symbiotic Behaviors}

For the convenience of the reader, we summarize here the main types of symbiotic behaviors for the considered cases of symbiosis, classifying the population dynamics with respect to the related symbiotic parameters.

\subsection{Dynamics under passive symbiosis}

The following types of population behavior can happen:

- Unbounded growth for

$$
0<b<\infty, \quad g>g_{c}(b)>0 .
$$

- Convergence to stationary states, if the initial conditions are inside the attraction basin, and unbounded growth, when the initial conditions are outside the attraction basin, which occurs for

$$
0<b<\infty, \quad 0<g<g_{c}(b) .
$$


- Convergence to stationary states for arbitrary initial conditions, when either

$$
b<0, \quad g>0,
$$

or

$$
b>0, \quad g<0 .
$$

- Convergence to stationary states in the presence of bistability, when

$$
b<0, \quad g<0 .
$$

\subsection{Dynamics under active symbiosis}

The following regimes of population dynamics exist:

- Unbounded growth if either

$$
b+g>\frac{1}{e}, \quad b>0, \quad g>0
$$

or

$$
b+g<\frac{1}{e}, \quad b>0, \quad g>0,
$$

and initial conditions are outside of the attraction basin.

- Dying out of the host species and unbounded proliferation of parasitic species, when either

$$
b<0, \quad g>\frac{1}{e}+|b|,
$$

or

$$
b>\frac{1}{e}+|g|, \quad g<0
$$

or

$$
b<0, \quad|b|<g<\frac{1}{e}+|b|,
$$

and initial conditions are outside of the attraction basin, or

$$
g<0, \quad|g|<b<\frac{1}{e}+|g|,
$$

and initial conditions are outside of the attraction basin.

- Convergence to stationary states for the initial conditions inside the attraction basin if

$$
b+g \leq \frac{1}{e} .
$$

\subsection{Dynamics under mixed symbiosis}

There can exist the following regimes of population dynamics:

- Unbounded growth when either

$$
0<b<\frac{1}{e}, \quad g>g_{c}(b)>0
$$

or

$$
b>\frac{1}{e}, \quad g \geq 0 .
$$

- Convergence to stationary states for the initial conditions inside the attraction basin and unbounded growth for the initial conditions outside the attraction basin, when

$$
0<b<\frac{1}{e}, \quad 0 \leq g<g_{c}(b) .
$$


- Convergence to stationary states for all initial conditions, if either

$$
b>\frac{1}{e}, \quad g<g_{c}(b)<0,
$$

or

$$
0<b<\frac{1}{e}, \quad g \leq 0
$$

or

$$
b \leq 0, \quad-\infty<g<\infty .
$$

- Everlasting oscillations under the condition

$$
b>\frac{1}{e}, \quad g_{c}(b)<g<0 .
$$

\section{Conclusion}

A novel approach to treating symbiotic relations between biological or social species has been suggested. The principal idea of the approach is the characterization of symbiotic relations of coexisting species through the carrying capacities of each other. Taking into account that the mutual influence can be quite strong, the carrying capacities are modeled by nonlinear functionals. We opt for the exponential form of this functional, since such a form naturally appears as an effective sum of a series, summed in a self-similar way, under the condition of semi-positivity.

We distinguish three variants of mutual influence, depending on the type of relations between the species. In the case of passive symbiosis, the mutual carrying capacities are influenced by other species without their direct interactions. In active symbiosis, the carrying capacities are influenced by interacting species. And mixed symbiosis describes the situation when the carrying capacity of one species is influenced by direct interactions, while that of the other species is not.

The approach allows us to describe all kinds of symbiosis, that is, mutualism, commensalism, and parasitism. The case of two symbiotic species is analyzed in detail. Depending on the symbiotic parameters, characterizing the destruction or creation of the mutual carrying capacities, that is, describing mutualistic or parasitic species, several dynamical regimes of coexistence are possible: unbounded growth of both populations, growth of one and the elimination of the other population, convergence to evolutionary stable states, and everlasting population oscillations.

The main difference of the described symbiosis with nonlinear functional carrying capacities, compared with the model of [Yukalov et al., 2012a, b] considered earlier of symbiosis with carrying capacities in the linear or bilinear approximations, is the absence of finite-time singularities and of an abrupt death of populations. The latter have appeared in the previous works [Yukalov et al., 2012a, b] owing to the artificial change of sign of the carrying capacities, when they were crossing zero. The nonlinear carrying capacities, employed in the present paper, are semi-positive defined, which ensures that the crossing-zero problem is avoided.

Among various bifurcations, when the dynamic regimes of the population evolution qualitatively changes, the most interesting ones are the two bifurcations occurring for the case of mixed symbiosis, with the coexistence of two parasitic species, when crossing the bifurcation line $g_{c}(b)$. One is the supercritical Hopf bifurcation, when a stable focus becomes unstable and a limit cycle arises. The other is the coalescence of a stable node with a saddle, with their disappearance and the appearance of a limit cycle. 


\section{References}

Ahmadjian, V. \& Paracer, S. [2000] An Introduction to Bilogical Associations (Oxford University, Oxford). Boucher, D. [1988] The Biology of Mutualism: Ecology and Evolution (Oxford University, New York).

Chen, G., Hill, D.J. \& Yu, X.H. [2003] Bifurcation Control: Theory and Applications (Springer, Berlin). Cobiaga, R, \$ Reartes, W. [2013] "A new approach in the search for periodic orbits", Int. J. Bifur. Chaos 23, 1350186.

Desroches, M., Guckenheimer, J., Krauskopf, B., Kuehn, C., Osinga, H. M. \& Wechselberger, M. [2012] "Mixed-mode oscillations with multiple time scales", SIAM Review 54, 211-288.

Douglas, A. E. [1994] Symbiotic Interactions (Oxford University, Oxford).

Gluzman, S. \& Yukalov, V. I. [1997] "Algebraic self-similar renormalization in the theory of critical phenomena", Phys. Rev. E 55, 3983-3999.

Gluzman, S. \& Yukalov, V. I. [1998a] "Resummation method for analyzing time series", Mod. Phys. Lett. $B$ 12, 61-74.

Gluzman, S. \& Yukalov, V. I. [1998b] "Renormalization group analysis of October market crashes", Mod. Phys. Lett. B 12, 75-84.

Gluzman, S. \& Yukalov, V. I. [1998c] "Booms and crashes in self-similar markets", Mod. Phys. Lett. B 12, $575-587$.

Gluzman, S., Sornette, D. \& Yukalov, V. I. [2003] "Reconstructing generalized exponential laws by selfsimilar exponential approximants", Int. J. Mod. Phys. C 14, 509-527.

Graedel, T. \& Allenby, B. [2003] Industrial Ecology (Prentice Hall, Englewood Cliffs).

Grossman, G. M. \& Helpman, E. [1991] Innovation and Growth in the Global Economy (MIT, Massachusetts).

Kuznetsov, Y. A. [1995] Elements of Applied Bifurcation Theory (Springer, Berlin).

Leonov, G. A. \& Kuznetsov, N. V. [2007] "Time-varying linearization and the Perron effect", Int. J. Bifur. Chaos 17, 1079-1107.

Leonov, G. A. \& Kuznetsov, N. V. [2013] "Hidden attractors in dynamical systems", Int. J. Bifur. Chaos 23, 1330002.

Lotka, A. J. [1925] Elements of Physical Biology (Williams and Wilkins, Baltimore).

Perc, M. \& Szolnoki, A. [2010] "Coevolutionary games - a mini review", Biosystems 99, 109-125.

Perc, M. \& Szolnoki, A. [2012] "Self-organization of punishment in structured populations", New J, Phys. 14, 043013.

Perc, M., Gomez-Gardenes, Szolnoki, A., Floria, L. M. \& Moreno, J. [2013] "Evolutionary dynamics of group interactions on structural populations: a review" J. Roy. Soc. Interface 10, 20120997.

Pongvuthithum, R. \& Likasiri, C. [2010] "Analytical discussions on species extinction in competitive communities due to habitat destruction", Ecol. Model. 221, 2634-2641.

Richard, R. R. [1993] National Innovation Systems: A Comparative Analysis (Oxford University, Oxford).

Szolnoki, A. \& Perc, M. [2013a] "Effectiveness of conditional punishment for the evolution of public cooperation", J. Theor. Biol. 325, 34-41.

Szolnoki, A. \& Perc, M. [2013b] "Information sharing promotes prosocial behavior", New J. Phys. 15, 053010 .

Sapp, J. [1994] Evolution by Association: A History of Symbiosis (Oxford University, Oxford).

Thompson, J. M., Stewart, H. B. \& Ueda, Y. [1994] "Safe, explosive, and dangerous bifurcations in dissipative dynamical systems", Phys. Rev. E 49, 1019-1027.

Townsend, C. R., Begon, M. \& Harper, J. D. [2002] Ecology: Individuals, Populations and Communities (Blackwell Science, Oxford).

Von Hippel, E. [1988] The Sources of Innovation (Oxford University, Oxford).

Yukalov, V. I. [1990] "Self-similar approximations for strongly interacting systems", Physica A 167, 833860.

Yukalov, V. I. [1991] "Method of self-similar approximations", J. Math. Phys. 32, 1235-1239.

Yukalov, V. I. [1992] "Stability conditions for method of self-similar approximations", J. Math. Phys. 33, $3994-4001$. 
Yukalov, V. I. \& Yukalova, E.P. [1996] "Temporal dynamics in perturbation theory", Physica A 225, $336-362$.

Yukalov, V. I. \& Gluzman, S. [1998] "Self-similar exponential approximants", Phys. Rev. E 58, 1359-1382.

Yukalov, V. I. \& Gluzman, S. [1999] "Weighted fixed points in self-similar analysis of time series", Int. J. Mod. Phys. B 13, 1463-1476.

Yukalov, V. I., Yukalova, E. P. \& Sornette, D. [2012a] "Modeling symbiosis by interactions through species carrying capacities", Physica D 241, 1270-1289.

Yukalov, V. I., Yukalova, E. P. \& Sornette, D. [2012b] "Extreme events in population dynamics with functional carrying capacity", Eur. Phys. J. Spec. Top. 205, 313-354.

Yukalov, V. I., Yukalova, E. P. \& Sornette, D. [2013] "Utility rate equations of group population dynamics in biological and social systems", PLOS One 8, e83225.

Yukalov, V. I., Yukalova, E. P. \& Sornette, D. [2014] "Population dynamics with nonlinear delayed carrying capacity", Int. J. Bifur. Chaos 24, 1450021. 

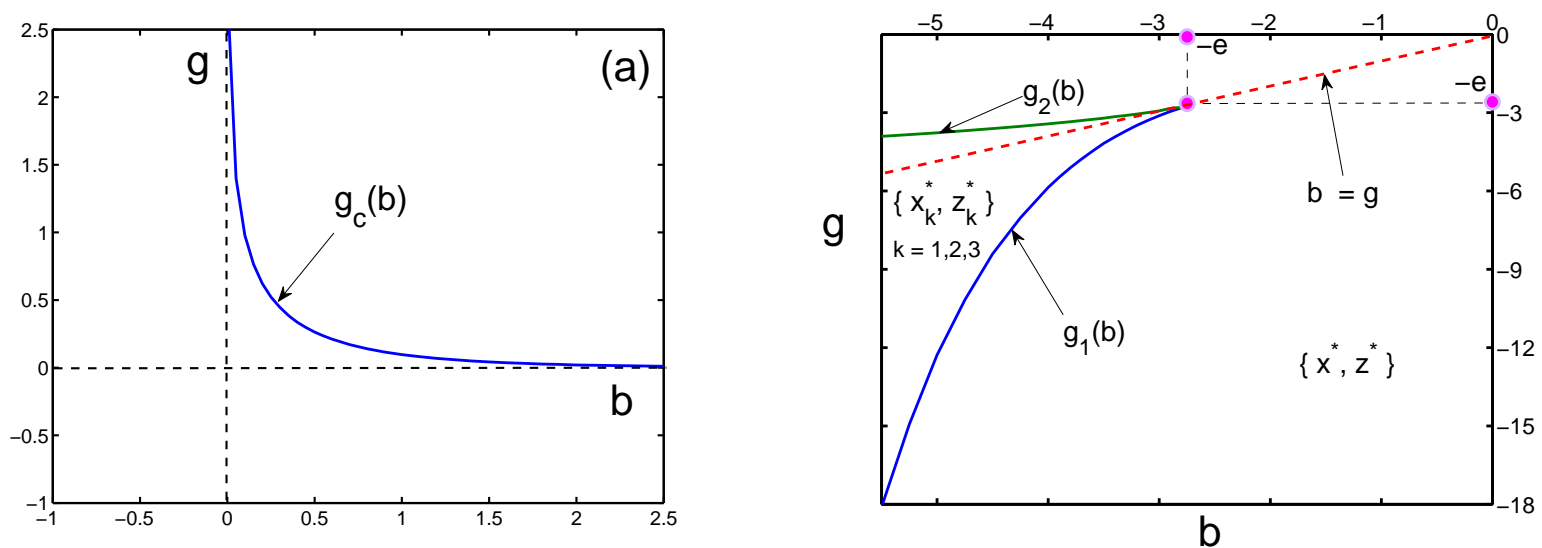

Fig. 1. (a) The regions of existence of stationary states for passive symbiosis in the plane $b-g$. When $b>0$ and $0<g<g_{c}(b)$, there exist two fixed points, but only one is stable. If $b \leq 0$ and $g>0$, or $b>0$ and $g \leq 0$, there exists a single fixed point that is stable. If $b, g<0$, there exist up to three fixed points, but not more then two of them are stable. (b) The region of fixed-point existence under mutual parasitism, when $b, g<0$. For either $-e<b<0$ and $g<0$, or for $b<-e$ and $g<g_{1}(b)$, or for $b<-e$ and $g_{2}(b)<g<0$, there exists only one fixed point that is stable. For $b<-e$ and $g_{1}(b)<g<g_{2}(b)$, there exist three fixed points, but only two of them are stable. 

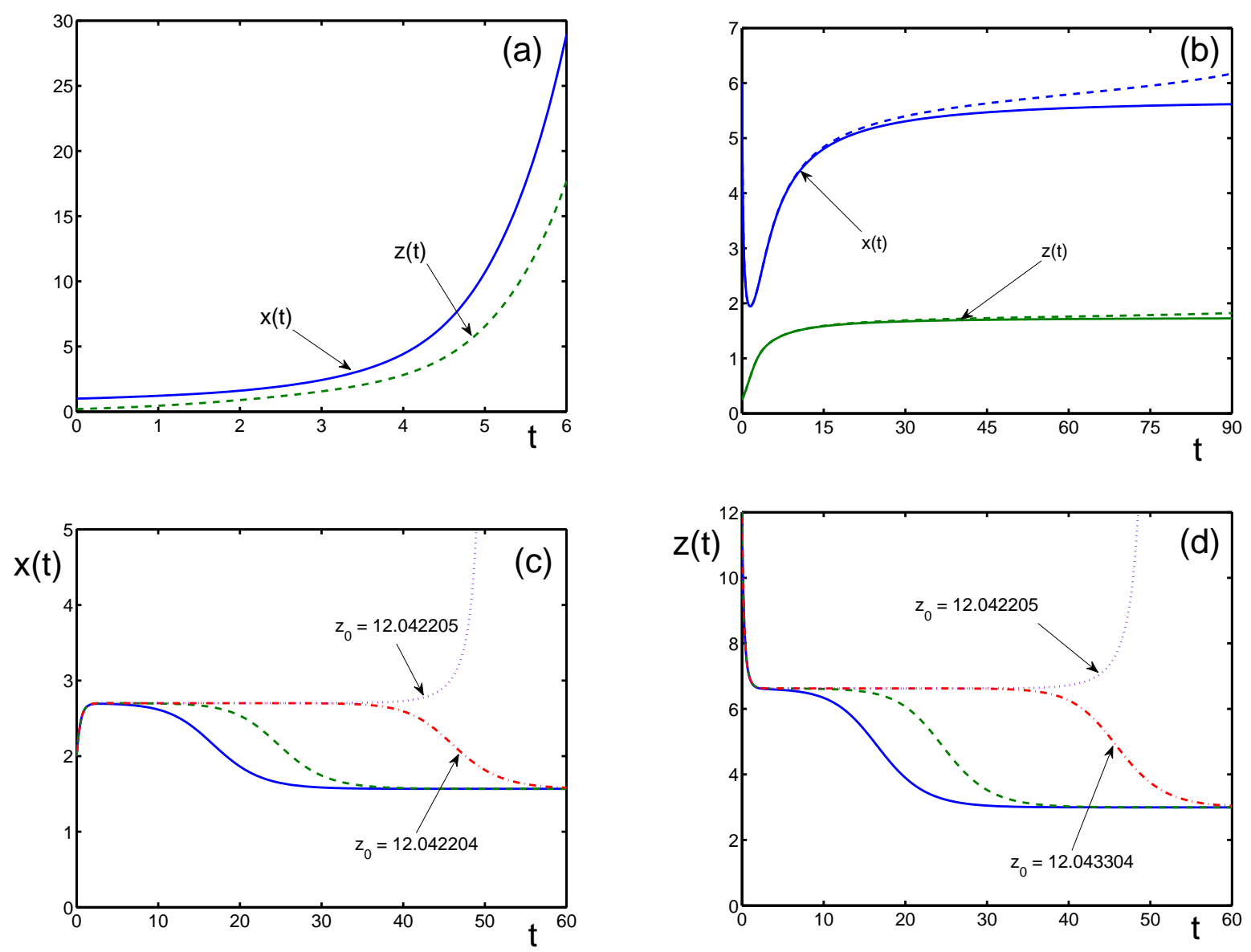

Fig. 2. Dynamics of populations in the case of mutualistic passive symbiosis for different symbiotic parameters $b>0$ and $g>0$, and different initial conditions: (a) For $b=1, g=0.5>g_{c}(b) \approx 0.0973$, and $x_{0}=1, z_{0}=0.2$, populations $x(t)$ (solid line) and $z(t)$ (dashed line) monotonically increase, as $t \rightarrow \infty$; (b) For the same $b=1$, as in Fig. 2a, with the initial conditions $x_{0}=6$ and $z_{0}=0.25$, for different $g$ taken slightly below and above the critical line $g=g_{c}(b)$. Below the critical line, when $g=0.0972<g_{c}=0.0973$, the population $x(t)$ tends to its stable fixed point $x^{*}=5.672$ and the population $z(t)$ tends monotonically from below to $z^{*}=1.735$. Both populations are shown by solid lines. Above the critical line, when $g=0.0976>g_{c}$, both populations, shown by dashed lines, tend to infinity, as $t \rightarrow \infty$; (c) Dynamics of the population $x(t)$ for the symbiotic parameters $b=0.15$ and $g=0.7<g_{c}=0.765$, with the initial condition $x_{0}=2$, but for different initial conditions $z_{0}$. The population $x(t)$ tends non-monotonically to its stable fixed point $x^{*}=1.567$, when $z_{0}$ is taken from the basin of attraction of this point: $x(t) \rightarrow x^{*}$ for $z_{0}=12$ (solid line), $z_{0}=12.04$ (dashed line), $z_{0}=12.042204$ (dashed-dotted line), and $x(t) \rightarrow \infty$, as $t \rightarrow \infty$, when $z_{0}=12.042205$ (dotted line); (d) Dynamics of the population $z(t)$ for the same parameters $b, g$ and the same initial conditions, as in Fig. 2c. The population converges to a stationary state, when the initial conditions are inside the attraction basin, and diverges, if the initial conditions are outside of this basin. 

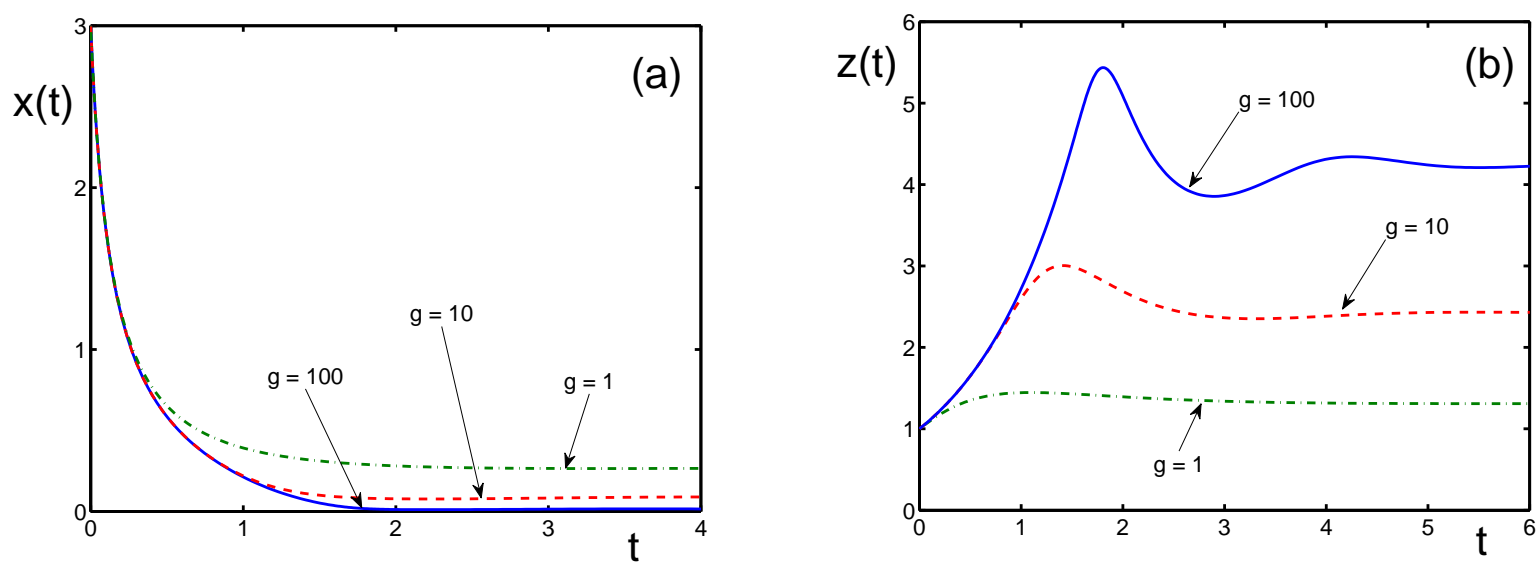

Fig. 3. Dynamics of populations under passive symbiosis with one parasitic species. Influence of the symbiotic parameter $g>0$ on the behaviour of the populations $x(t), z(t)$, for the fixed $b=-1$, and the initial conditions $x_{0}=3$ and $z_{0}=1$. Different lines correspond to the varying parameter $g$ : For $g=1$ (dashed-dotted line) the stable fixed point is $\left\{x^{*}=0.270, z^{*}=1.31\right\}$; for $g=10$ (dashed line), the fixed point is $\left\{x^{*}=0.089, z^{*}=2.42\right\}$; and for $g=100$ (solid line), the stationary point is $\left\{x^{*}=0.014, z^{*}=4.24\right\}$. (a) Population $x(t)$ tends monotonically from above to the corresponding stable stationary state, as $t \rightarrow \infty$; (b) Population $z(t)$ tends non-monotonically from below to the corresponding stable stationary state, as $t \rightarrow \infty$. 

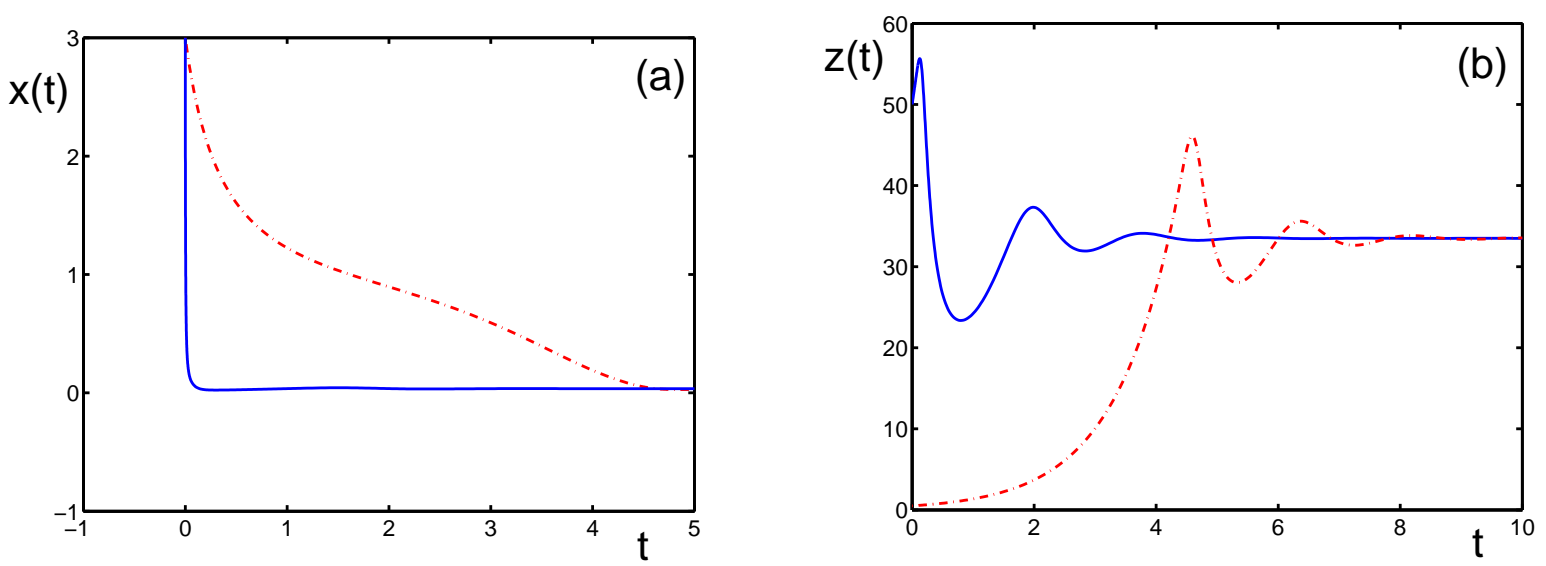

Fig. 4. Dynamics of populations under passive symbiosis with one parasitic species. Influence of the initial condition $z_{0}$ on the behavior of solutions for the fixed $b=-0.1, g=100$, and $x_{0}=3$. The corresponding stable fixed point is $\left\{x^{*}=0.0335, z^{*}=\right.$ 33.49\}. (a) Population $x(t)$ tends monotonically, as $t \rightarrow \infty$, from above to the stable stationary state $x^{*}$ for $z_{0}=50$ (solid line) and for $z_{0}=0.5$ (dashed line); (b) Population $z(t)$ tends, as $t \rightarrow \infty$, non-monotonically from above to the stable stationary state $z^{*}$ for $z_{0}=50$ (solid line) and non-monotonically from below to $z^{*}$ for $z_{0}=0.5$ (dashed line). 

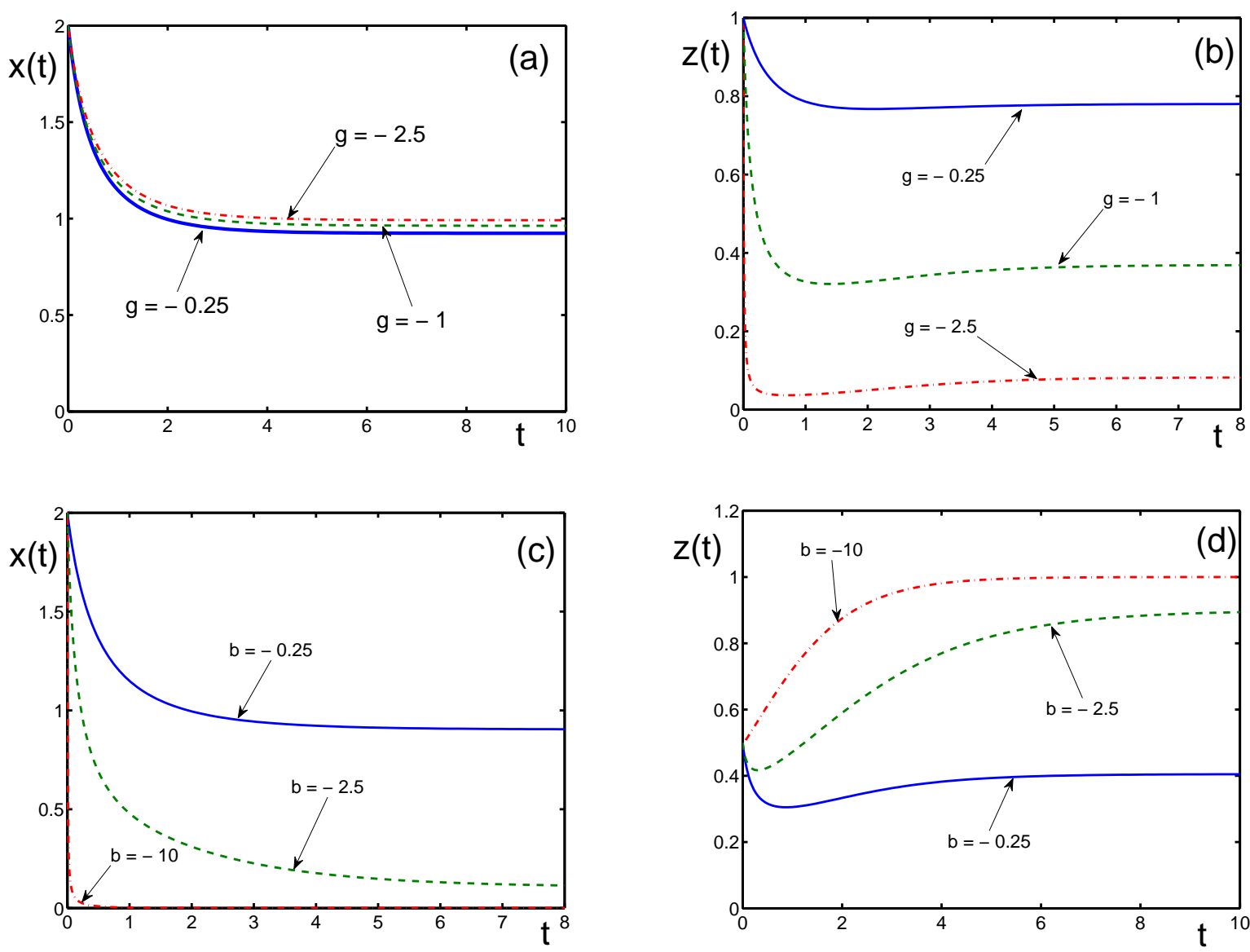

Fig. 5. Dynamics of populations under passive symbiosis with two parasitic species. Influence of the symbiotic parameters $b<0$ and $g<0$ under the fixed initial conditions $\left\{x_{0}=2, z_{0}=1\right\}$. (a) Dynamics of $x(t)$ for $b=-0.1$ and different parameters $g$. The population $x(t)$ monotonically tends to its stationary state: $\left\{x^{*}=0.924, z^{*}=0.794\right\}$ for $g=-0.25$ (solid line); $\left\{x^{*}=0.963, z^{*}=0.382\right\}$ for $g=-1$ (dashed line); and $\left\{x^{*}=0.992, z^{*}=0.084\right\}$ for $g=-2.5$ (dashed-dotted line). (b) Dynamics of $z(t)$ for the same, as above, parameters $b=-0.1$ and different $g$. Population $z(t)$ monotonically, or non-monotonically, from above tends to the corresponding stationary states. (c) Dynamics of $x(t)$ for $g=-1$ and different parameters $b$. The population $x(t)$ monotonically tends from above to its stationary state: $\left\{x^{*}=0.904, z^{*}=0.405\right\}$ for $b=-0.25$ (solid line); $\left\{x^{*}=0.105, z^{*}=0.899\right\}$ for $b=-2.5$ (dashed line); and $\left\{x^{*}=0.454 \times 10^{-4}, z^{*}=0.99996\right\}$ for $b=-10$ (dashed-dotted line). (d) Dynamics of $z(t)$ for the same, as in Fig. 5 c, parameters $g=-1$ and different $b$. Population $z(t)$ converges to the corresponding stationary states, either monotonically or non-monotonically. 

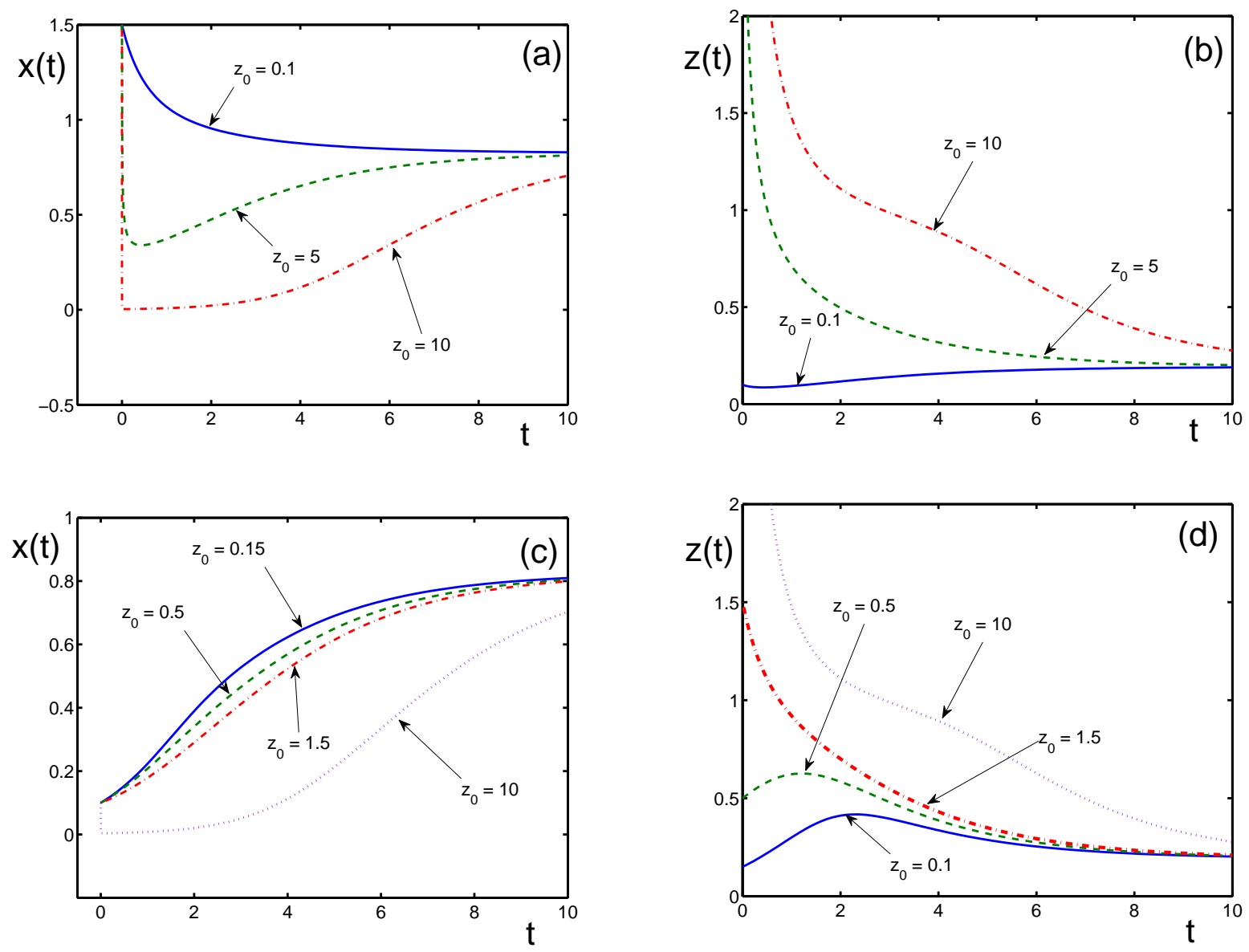

Fig. 6. Dynamics of populations under passive symbiosis with two parasitic species. Influence of initial conditions $\left\{x_{0}, z_{0}\right\}$ on the behaviour of the parasitic populations with $b=-1$ and $g=-2$. The corresponding stable fixed point is $\left\{x^{*}=\right.$ $\left.0.825, z^{*}=0.192\right\}$. (a) Dynamics of $x(t)$ for $x_{0}=1.5>x^{*}$ and different $z_{0}$. Population $x(t)$ converges to the stationary state $x^{*}$ : monotonically for $z_{0}=0.1<z^{*}$ (solid line); non-monotonically for $z_{0}=5$ (dashed line); and non-monotonically for $z_{0}=10>z^{*}$ (dashed-dotted line); (b) Dynamics of $z(t)$ for the same, as in Fig. 6a, initial condition $x_{0}=1.5$ and different $z_{0}$. Population $z(t)$ tends to the stationary state $z^{*}$ : non-monotonically for $z_{0}=0.1$ (solid line); monotonically for $z_{0}=5$ (dashed line); and monotonically for $z_{0}=10$ (dashed-dotted line). (c) Dynamics of $x(t)$ for $x_{0}=0.1<x^{*}$ and different $z_{0}$. Population $x(t)$ converges to the stationary state $x^{*}$ : monotonically for $z_{0}=0.15$ (solid line); for $z_{0}=0.5$ (dashed line); and $z_{0}=1.5$ (dashed-dotted line); while non-monotonically for $z_{0}=10$ (dotted line). (d) Dynamics of $z(t)$ for the same, as in Fig. $6 \mathrm{c}$, initial condition $x_{0}=0.1$ and different $z_{0}$. Population $z(t)$ tends to the stationary state $z^{*}$ : non-monotonically for $z_{0}=0.1$ (solid line); and $z_{0}=0.5$ (dashed line); but monotonically for $z_{0}=1.5$ (dashed-dotted line); and $z_{0}=10$ (dotted line). 

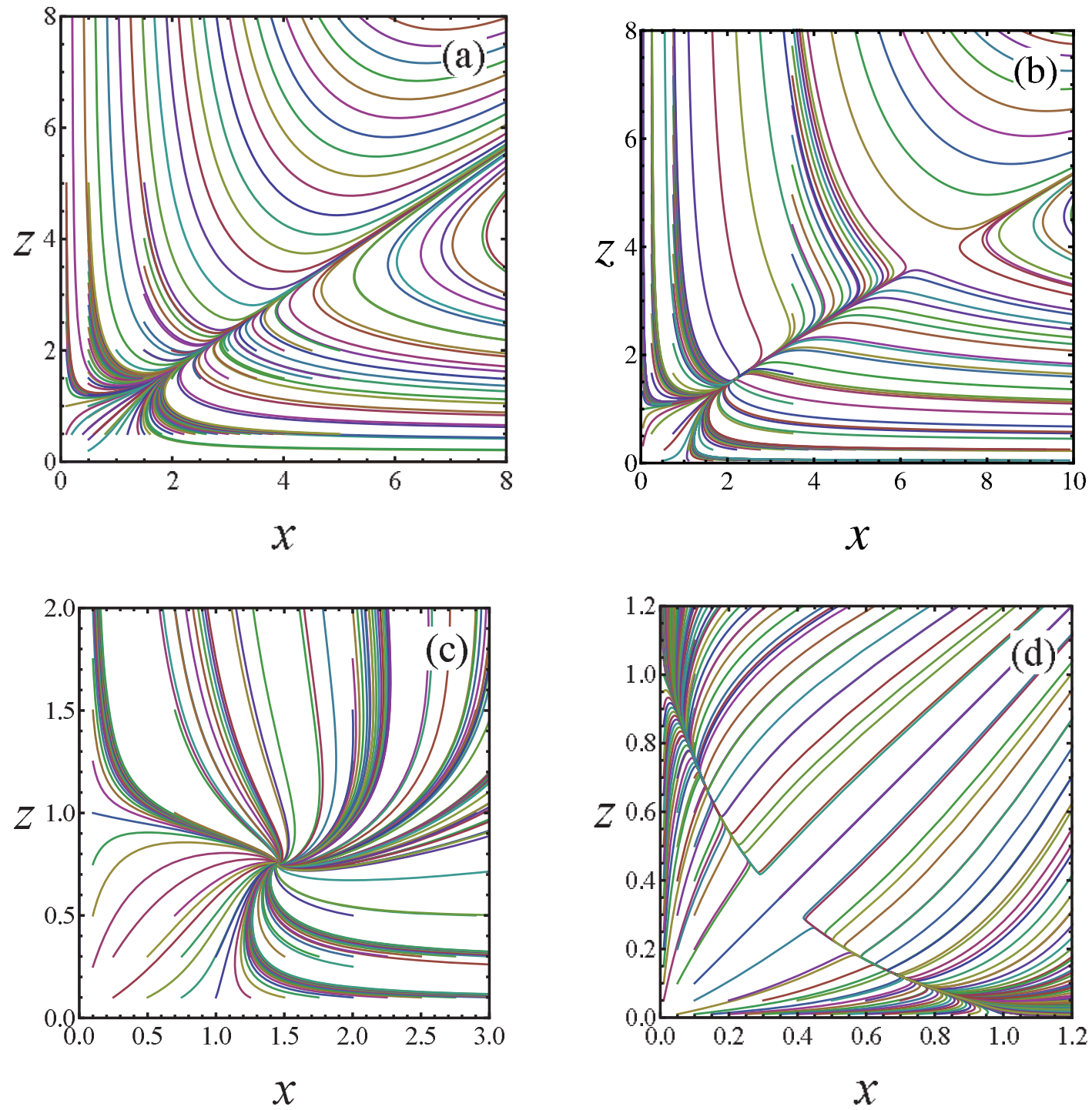

Fig. 7. Phase portrait for passive symbiosis, with the symbiotic parameters $b$ and $g$ in qualitatively different regions: (a) Phase portrait for $b=0.5$ and $g=0.3$, where $g>g_{c}(b) \simeq 0.263$. The parameters are in the region, where no fixed points exist for the system of equations (13). (b) Phase portrait for $b=0.5$ and $0<g=0.2<g_{c}(b) \simeq 0.263$. The parameters are in the region of $b, g$, where two fixed points exist. The first fixed point $\left\{x_{1}^{*}=2.16019, z_{1}^{*}=1.54039\right\}$ is a stable node, with the Lyapunov exponents $\lambda_{1}=-1.57685, \lambda_{2}=-0.42315$, while the second point $\left\{x_{2}^{*}=6.66791, z_{2}^{*}=3.7946\right\}$ is a saddle, with the Lyapunov exponents $\lambda_{1}=-2.59067, \lambda_{2}=0.59067$. (c) Phase portrait for $b=0.5$ and $g=-0.2$. The parameters are in the region of $b, g$, where only one fixed point exists, which is a stable focus $\left\{x^{*}=1.45336, z_{1}^{*}=0.74776\right\}$, with the characteristic exponents $\lambda_{1}=-1-0.32966 i, \lambda_{2}=-1+0.32966 i$. (d) Phase portrait for $b=g=-3$. The parameters are in the bistability region of $b, g$, where three fixed points exist, two of them being stable and one, unstable. Here, the first stable fixed point is the node $\left\{x_{1}^{*}=0.88474, z_{1}^{*}=0.13612\right\}$, with the Lyapunov exponents $\lambda_{1}=-1.90242, \lambda_{2}=-0.0975818$. The second stable fixed point, due to symmetry, is the node $\left\{x_{2}^{*}=z_{1}^{*}, z_{2}^{*}=x_{1}^{*}\right\}$, with the same Lyapunov exponents. And the third fixed point $\left\{x_{3}^{*}=z_{3}^{*}=0.34997\right\}$ is the saddle, with the Lyapunov exponents $\lambda_{1}=-2.04991, \lambda_{2}=0.0499089$. 


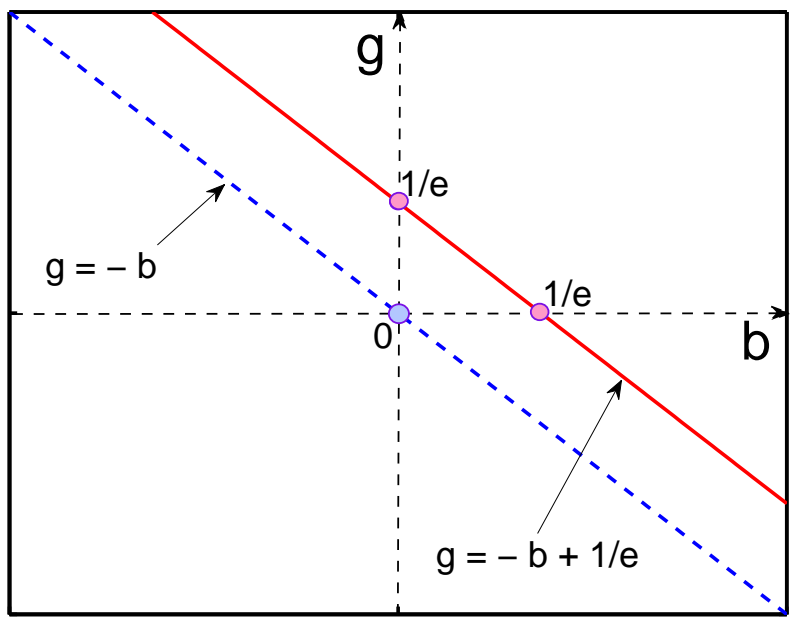

Fig. 8. The regions of existence of the fixed-point for Eqs. (16) in the plane $b-g$. When $b+g>1 / e$, then there are no fixed points. If $0<b+g<1 / e$, then there exist two fixed points, one of them being stable and another, unstable. If $b+g \leq 0$, then there exists a single fixed point that is stable. 

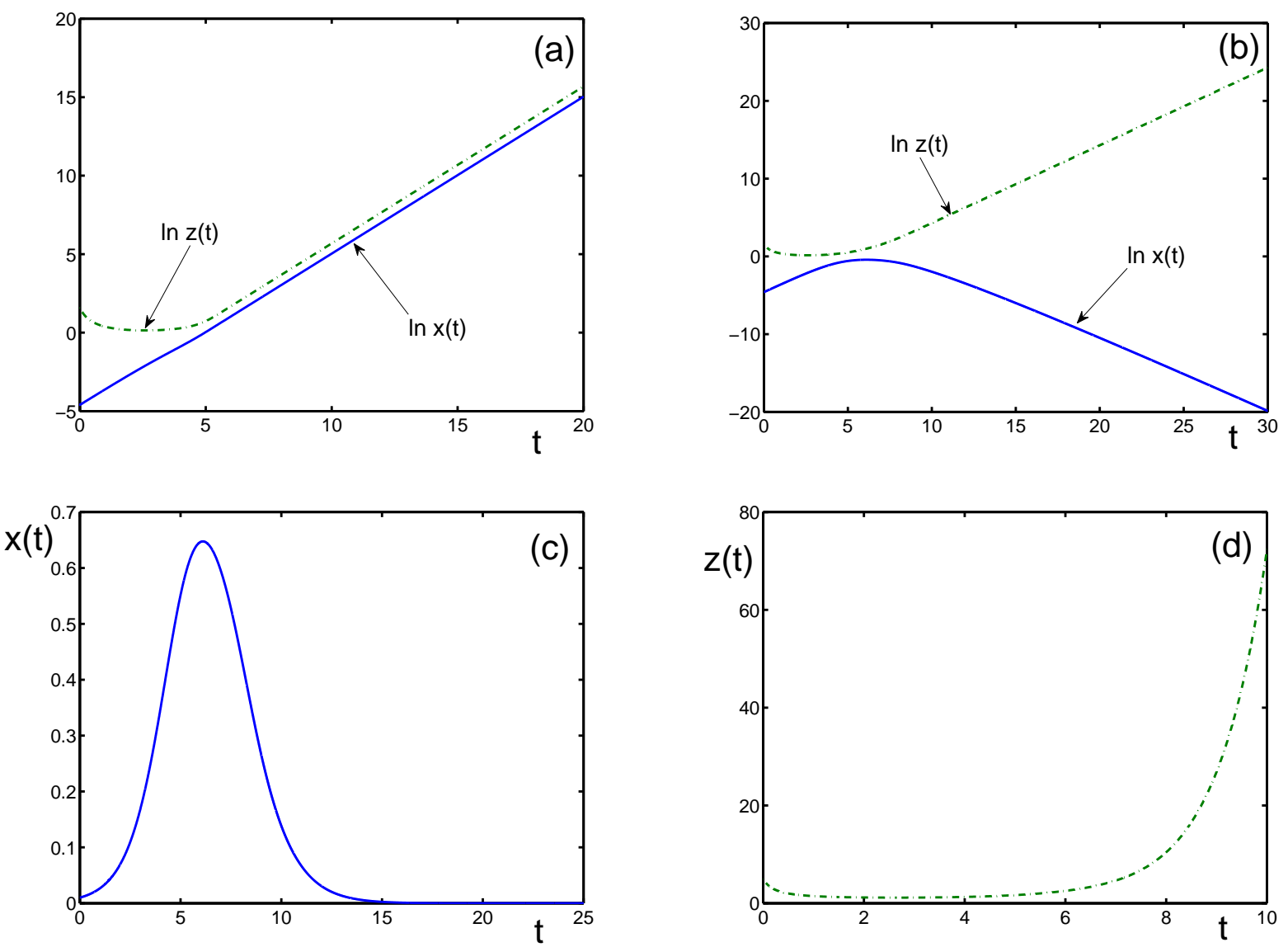

Fig. 9. Dynamics of populations under $b+g>1 / e$, with the initial conditions $x_{0}=0.01$ and $z_{0}=5$ for different symbiotic parameters: (a) Logarithmic behavior of mutualistic populations for $b=2$ and $g=1$. Both populations grow, as $t \rightarrow \infty$, with $\ln x(t) \rightarrow \infty$ increasing monotonically, while $\ln z(t) \rightarrow \infty$, non-monotonically. (b) Logarithmic behavior of the populations, when one of them is parasitic, for $b=-0.5$ and $g=1$. At $t \rightarrow \infty$, the host population dies out, $\ln x(t) \rightarrow-\infty$, that is, $x(t) \rightarrow 0$, and the parasitic population non-monotonically increases, $\ln z(t) \rightarrow \infty$. (c) Dynamics of the host population $x(t)$, for the same parameters $b=-0.5$ and $g=1$, demonstrating that $x(t) \rightarrow 0$ dies out, as $t \rightarrow \infty$, in a non-monotonic way. (d) Dynamics of the parasitic population $z(t)$, for the same parameters $b=-0.5$ and $g=1$, showing the details of the non-monotonic increase of $z(t) \rightarrow \infty$, as $t \rightarrow \infty$. 

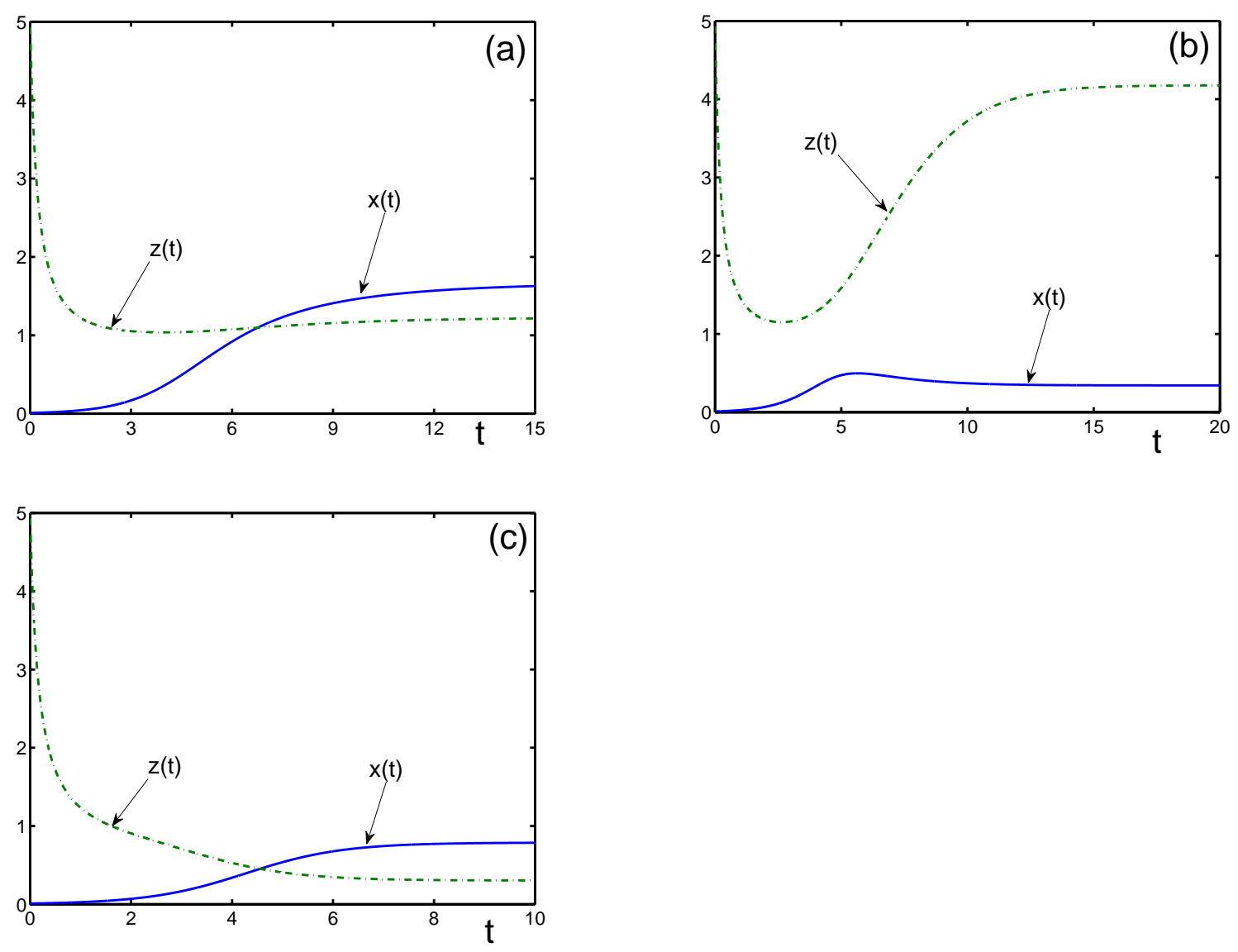

Fig. 10. Population dynamics for $0<b+g<1 / e$, with the initial conditions $x_{0}=0.01$ and $z_{0}=5$. (a) For $b=0.25$ and $g=0.1$, the population $x(t) \rightarrow x^{*}=1.668$ monotonically from below, and population $z(t) \rightarrow z^{*}=z^{*}=1.227$ nonmonotonically from above. (b) For $b=-0.75$ and $g=1$, the population $x(t) \rightarrow x^{*}=0.342$, nonmonotonically from below, and population $z(t) \rightarrow z^{*}=4.177$, non-monotonically from above. (c) For $b=-1$ and $g=-5$, the population $x(t) \rightarrow x^{*}=0.788$, monotonically from below, and population $z(t) \rightarrow z^{*}=0.303$ monotonically from above. 

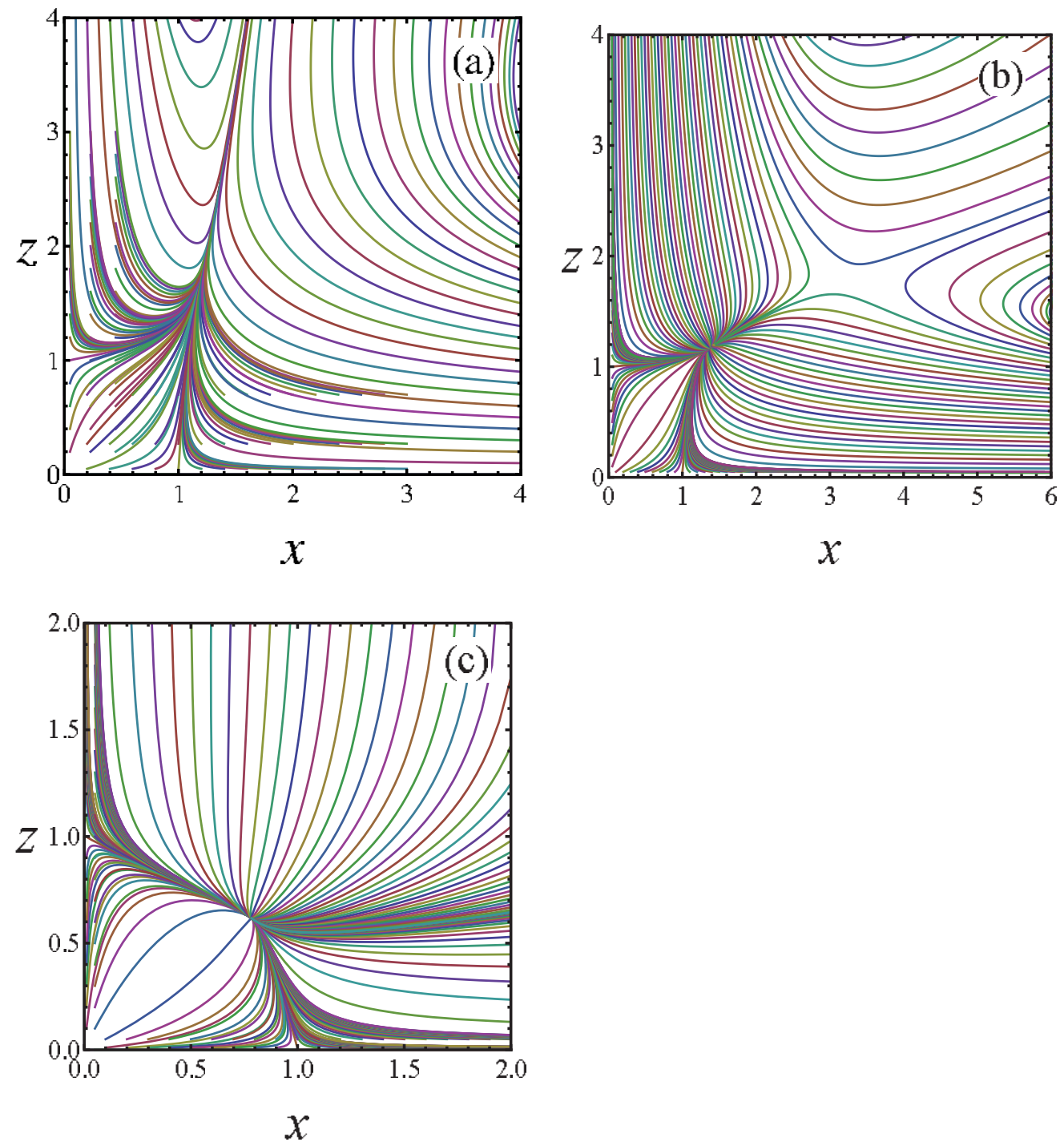

Fig. 11. Phase portrait for active symbiosis, with the symbiosis parameters $b$ and $g$ from qualitatively different regions: (a) Phase portrait for $b=0.1$ and $g=0.3$, where $b+g>1 / e$. The parameters are in the region, where there are no fixed points. (b) Phase portrait for $b=0.2$ and $g=0.1$, with $0<b+g_{c}(b)<1 / e$. The parameters are in the region of $b, g$, where two fixed points exist. The first fixed point $\left\{x_{1}^{*}=1.38579, z_{1}^{*}=1.17719\right\}$ is the stable node, with the Lyapunov exponents $\lambda_{1}=-1$, $\lambda_{2}=-1.72586$, and the second point $\left\{x_{2}^{*}=3.27906, z_{2}^{*}=1.81082\right\}$ is a saddle, with the Lyapunov exponents $\lambda_{1}=-1$, $\lambda_{2}=0.781337$. (c) Phase portrait for $b=-0.5$ and $g=-1$. The parameters are in the region of $b, g$, where only one fixed point exists, which is the stable node $\left\{x^{*}=0.78509, z^{*}=0.61637\right\}$, with the Lyapunov exponents $\lambda_{1}=-1, \lambda_{2}=-1.72586$. 


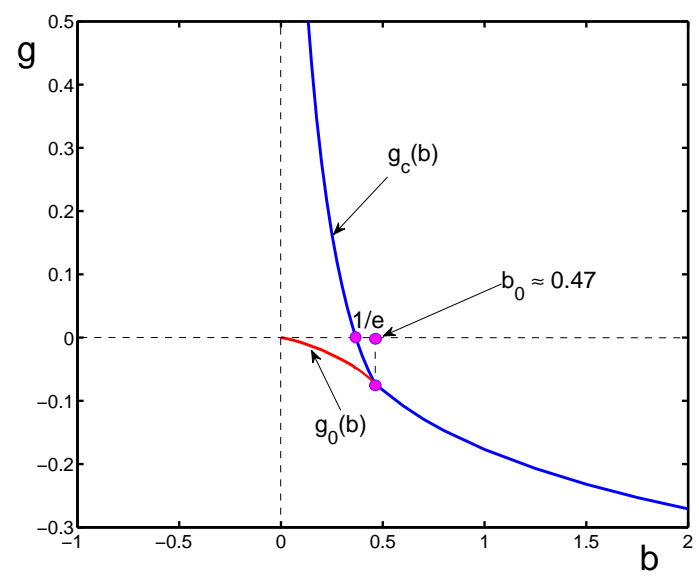

Fig. 12. Regions of existence of the fixed-point for mixed symbiosis. For either $0<b<1 / e$ and $g>g_{c}(b)$, or $b>1 / e$ and $g \geq 0$, Eqs. (21) do not have solutions, hence, there are no fixed points. For $0<b<1 / e$ and $0<g<g_{c}(b)$, Eqs. (21) have two solutions, but only one fixed point is stable. For $b>1 / e$ and $g_{c}(b)<g<0$, there exists one unstable fixed point. When either $0<b<1 / e$ and $g_{0}(b)<g<0$, or $1 / e<b<b_{0} \approx 0.47$ and $g_{0}(b)<g<g_{c}(b)$, then there are three fixed points, but only one of them is stable. When either $0<b<b_{0}$ and $g<g_{0}(b)$, or $b \geq b_{0}$ and $g<g_{c}(b)$, then there exists a single fixed point that is stable. When $b \leq 0$ and $g \in(-\infty, \infty)$, then the unique solution to Eqs. (21) is a stable fixed point. 

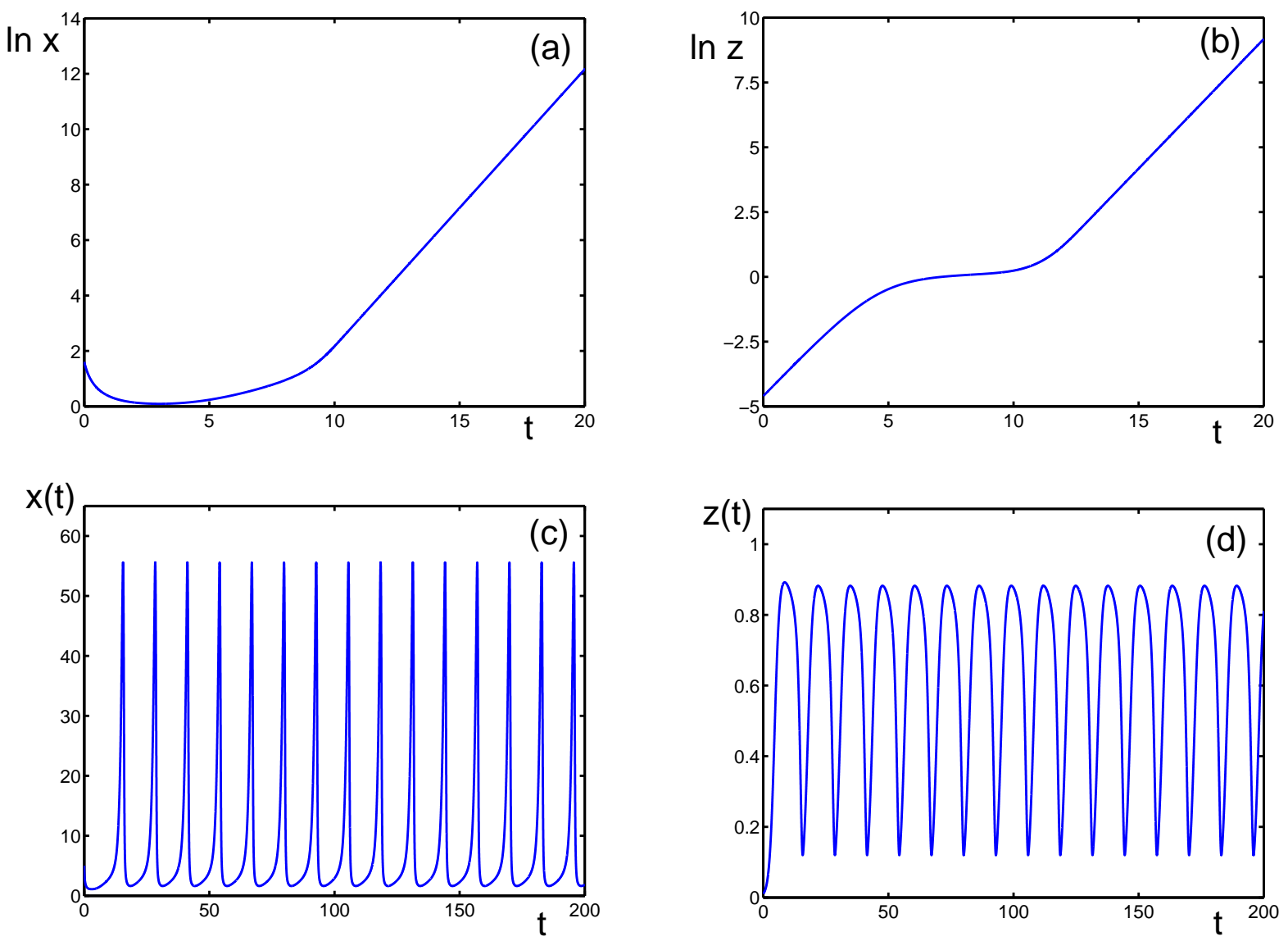

Fig. 13. Dynamics of populations under mixed symbiosis for the symbiotic parameters, when unbounded growth changes to everlasting oscillations. The symbiotic parameter of the active species is fixed, $b=0.5>1 / e$, while that of the passive species varies. For the given $b$, the change of the behavior occurs on the line $g=0$ that is higher than the Hopf bifurcation point $g_{c}(b)=-0.083065$. The initial conditions are $\left\{x_{0}=5, z_{0}=0.01\right\}$. (a) Logarithmic behaviour of the active population $x(t)$ for $g=0.05$, where there are no fixed points. (b) Logarithmic behaviour of the passive population $z(t)$ for $g=0.05$. (c) Active species population $x(t)$ oscillates without convergence for $g=-0.05>g_{c}(b)=-0.083065$. (d) Passive species population $z(t)$ oscillates without convergence for the same $g=-0.05$. 

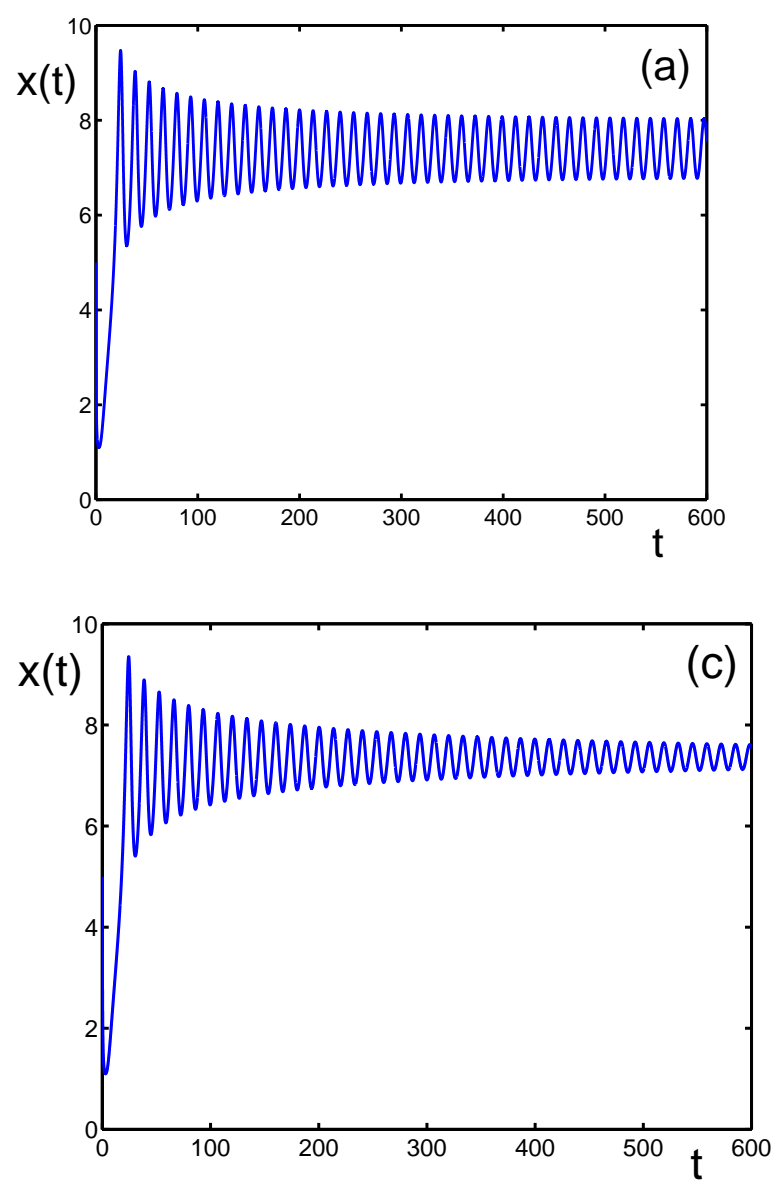
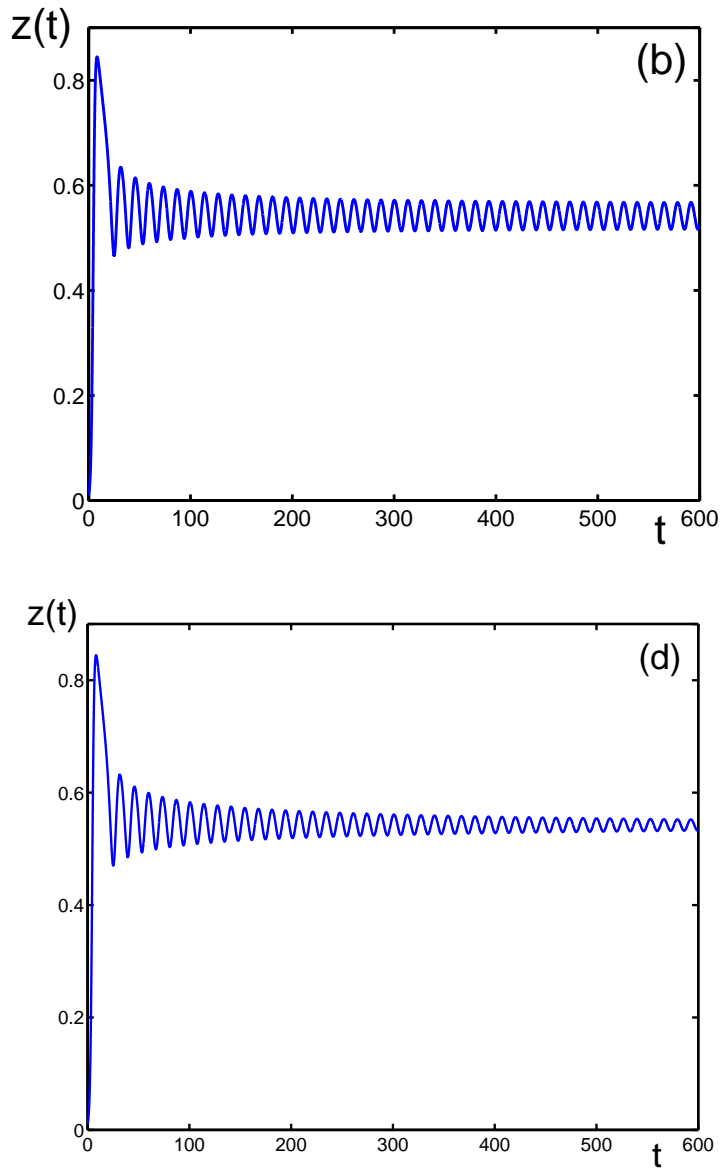

Fig. 14. Dynamics of populations under mixed symbiosis for the symbiotic parameters, when everlasting oscillations change to the oscillating convergence to a a stable focus. The symbiotic parameter of the active species is fixed, $b=0.5>1 / e$, with the Hopf bifurcation point $g_{c}(b)=-0.083065$. The initial conditions are the same as in Fig. $13,\left\{x_{0}=5, z_{0}=0.01\right\}$. (a) Active species population $x(t)$ oscillates with a small amplitude, as $t \rightarrow \infty$, when $g=-0.083>g_{c}(b)$ is slightly larger than the Hopf bifurcation point. The characteristic exponents for the unstable focus are $\lambda_{1}=0.0018+0.479 i$ and $\lambda_{2}=\lambda_{1}^{*}$. (b) Passive species population $z(t)$ oscillates, as $t \rightarrow \infty$, when $g=-0.083>g_{c}(b)$ is slightly larger than the Hopf bifurcation point. (c) Active species population $x(t)$ converges with oscillations to its stable stationary state $x^{*}=7.368$, when $g=-0.0831<g_{c}(b)$ is slightly lower than the Hopf bifurcation point. The characteristic exponents for the stable focus are $\lambda_{1}=-0.0014+0.475 i$ and $\lambda_{2}=\lambda_{1}^{*}$. (d) Passive species population $z(t)$ converges oscillating to its stable stationary state $z^{*}=0.542$, when $g=-0.0831<g_{c}(b)$ is slightly lower than the Hopf bifurcation point. 

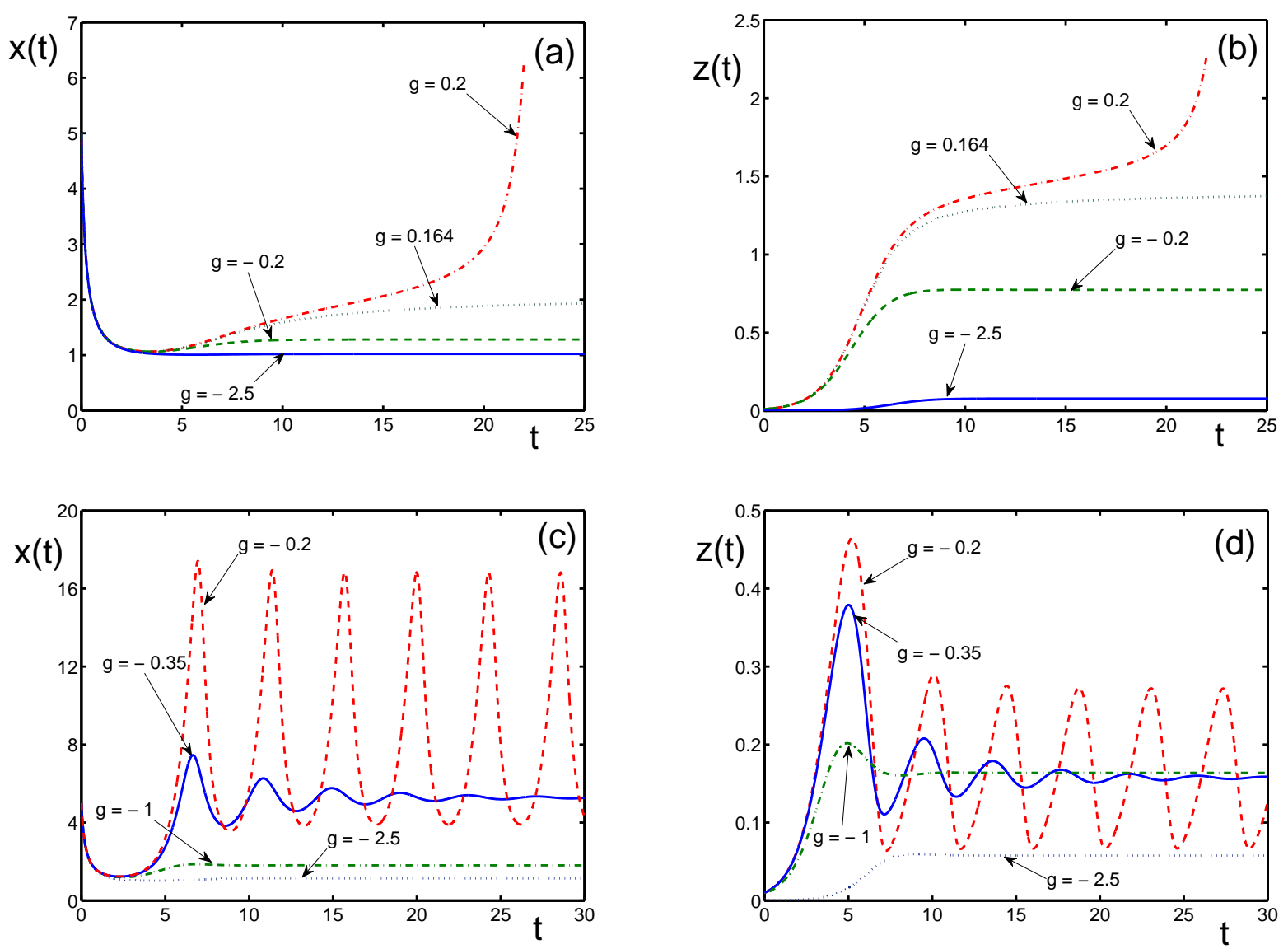

Fig. 15. Population dynamics under mixed symbiosis for varying symbiotic parameters, with the initial conditions $\left\{x_{0}=\right.$ $\left.5, z_{0}=0.01\right\}$. (a) Active species population dynamics for the fixed $b=0.25<1 / e$, when $g_{c}(b)=0.1645$ and $g_{0}(b)=-0.026564$, for different passive species symbiotic parameters: $g=-2.5<g_{0}(b)$ (solid line), when the population $x(t)$ tends to $x^{*}=1.02$; $g=-0.2$ (dashed line), when the population $x(t) \rightarrow x^{*}=1.28 ; 0<g=0.164<g_{c}(b)$ (dotted line), when the population $x(t) \rightarrow x^{*}=2.03$; and $g=0.2>g_{c}(b)$ (dashed-dotted line), when $x(t) \rightarrow \infty$, as $t \rightarrow \infty$. (b) Passive species population dynamics for $b=0.25$ and the same initial conditions, with the varying symbiotic parameter $g$ of the passive species: $g=-2.5$ (solid line), when $z(t) \rightarrow z^{*}=0.0781 ; g=-0.2$ (dashed line), when $z(t) \rightarrow z^{*}=0.774 ; g=0.164$ (dotted line), when $z(t) \rightarrow z^{*}=1.39$; and $g=0.2>g_{c}(b)$ (dashed-dotted line), when $x(t) \rightarrow \infty$, as $t \rightarrow \infty$. (c) Active species population dynamics under the fixed $b=2>b_{0} \approx 0.47$, with $g_{c}(b)=-0.271$, for different $g: g=-2.5$ (dotted line), when $x(t) \rightarrow x^{*}=1.14$; $g=-1$ (dashed-dotted line), with $x(t) \rightarrow x^{*}=1.81 ; g=-0.35<g_{c}(b)$ (solid line), when $x(t) \rightarrow x^{*}=5.28$ with a few oscillations; and $g=-0.2>g_{c}(b)$ (dashed line), when $x(t)$ oscillates without convergence, as $t \rightarrow \infty$. (d) Passive species population dynamics for $b=2$ and the same initial conditions, with the varying symbiotic parameter $g: g=-2.5$ (dotted line), when $z(t) \rightarrow z^{*}=0.0577 ; g=-1$ (dashed-dotted line), with $z(t) \rightarrow z^{*}=0.164 ; g=-0.35<g_{c}(b)$ (solid line), when $z(t) \rightarrow z^{*}=0.157$ with a few oscillations, and $g=-0.2>g_{c}(b)$ (dashed line), with $z(t)$ oscillating without convergence, as $t \rightarrow \infty$. 

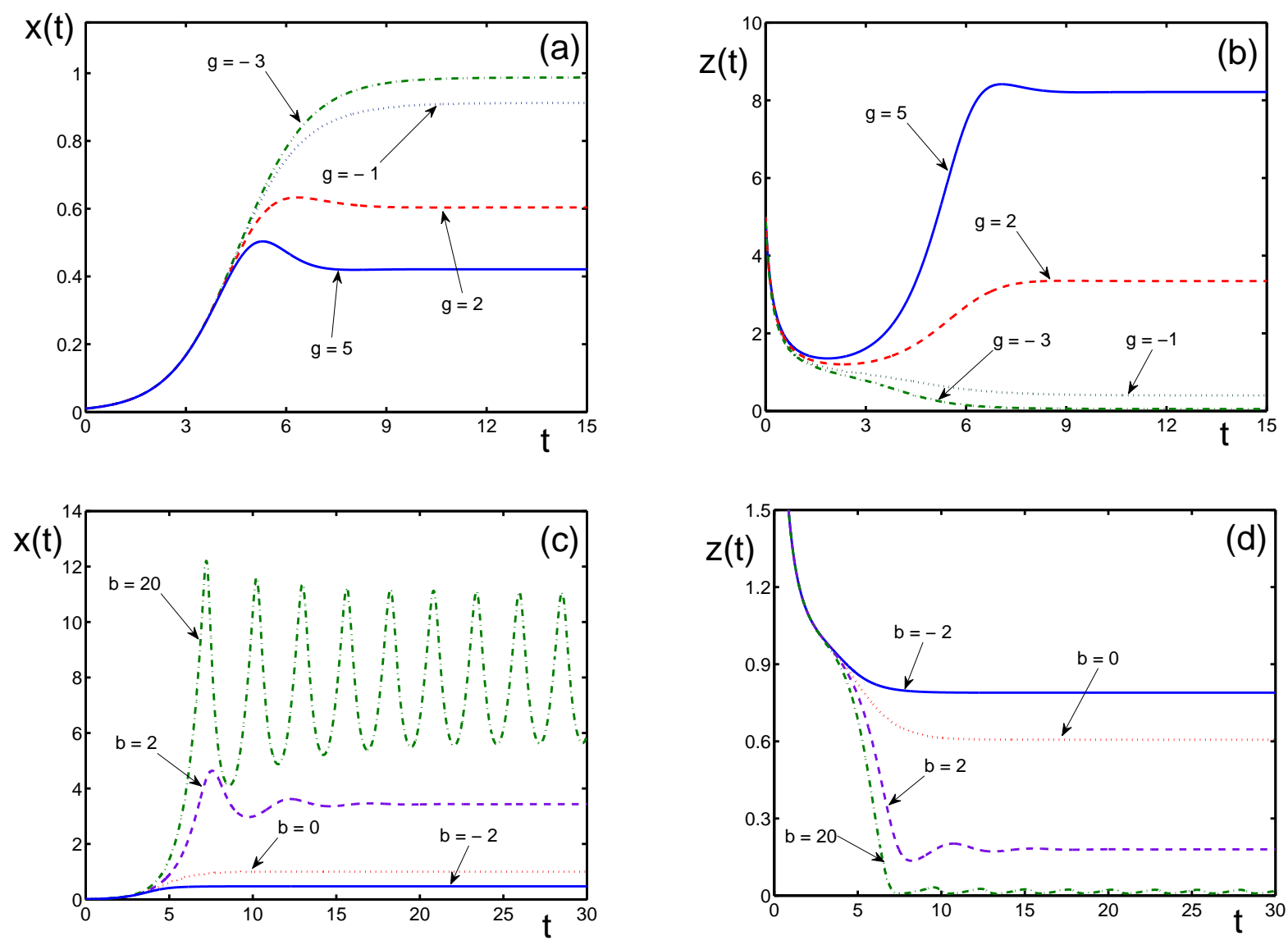

Fig. 16. Population dynamics under mixed symbiosis for varying symbiotic parameters, with the initial conditions $\left\{x_{0}=\right.$ $\left.0.01, z_{0}=5\right\}$. (a) Active species population dynamics under the fixed $b=-0.25$ and different $g: g=5$ (solid line), when $x(t) \rightarrow x^{*}=0.421 ; g=2$ (dashed line), with $x(t) \rightarrow x^{*}=0.604 ; g=-1$ (dotted line), when $x(t) \rightarrow x^{*}=0.912 ;$ and $g=-2$ (dashed-dotted line), with $x(t) \rightarrow x^{*}=0.987$. (b) Passive species population dynamics under the fixed $b=-0.25$ and different $g: g=5$ (solid line), with $z(t) \rightarrow z^{*}=8.21$ non-monotonically from below; $g=2$ (dashed line), when $z(t) \rightarrow z^{*}=3.34$ non-monotonically from above; $g=-1$ (dotted line), when $z(t) \rightarrow z^{*}=0.402$; and $g=-2$ (dashed-dotted line ), when $z(t) \rightarrow z^{*}=0.0517$ monotonically from above. (c) Active species population dynamics under the fixed $g=-0.5$ and different $b: b=-2$ (solid line), when $x(t) \rightarrow x^{*}=0.474 ; b=0$ (dotted line), with $x(t) \rightarrow x^{*}=1$ monotonically from below; $b=2$ (dashed line), when $g_{c}(b)=-0.271>g$, and $x(t) \rightarrow x^{*}=3.43$ with a few oscillations; $b=20$ (dashed-dotted line), when $g_{c}(b)=-0.5823<g<0$, and solution $x(t)$ oscillates without convergence, as $t \rightarrow \infty$. (d) Passive species population dynamics under the fixed $g=-0.5$ and different $b: b=-2$ (solid line), when $z(t) \rightarrow z^{*}=0.789 ; b=0$ (dotted line), with $z(t) \rightarrow z^{*}=0.607$ monotonically from above; $b=2$ (dashed line), when $z(t) \rightarrow z^{*}=0.1796$ with a few oscillations; and $b=20$ (dashed-dotted line), when $z(t)$ oscillates without convergence, as $t \rightarrow \infty$. 

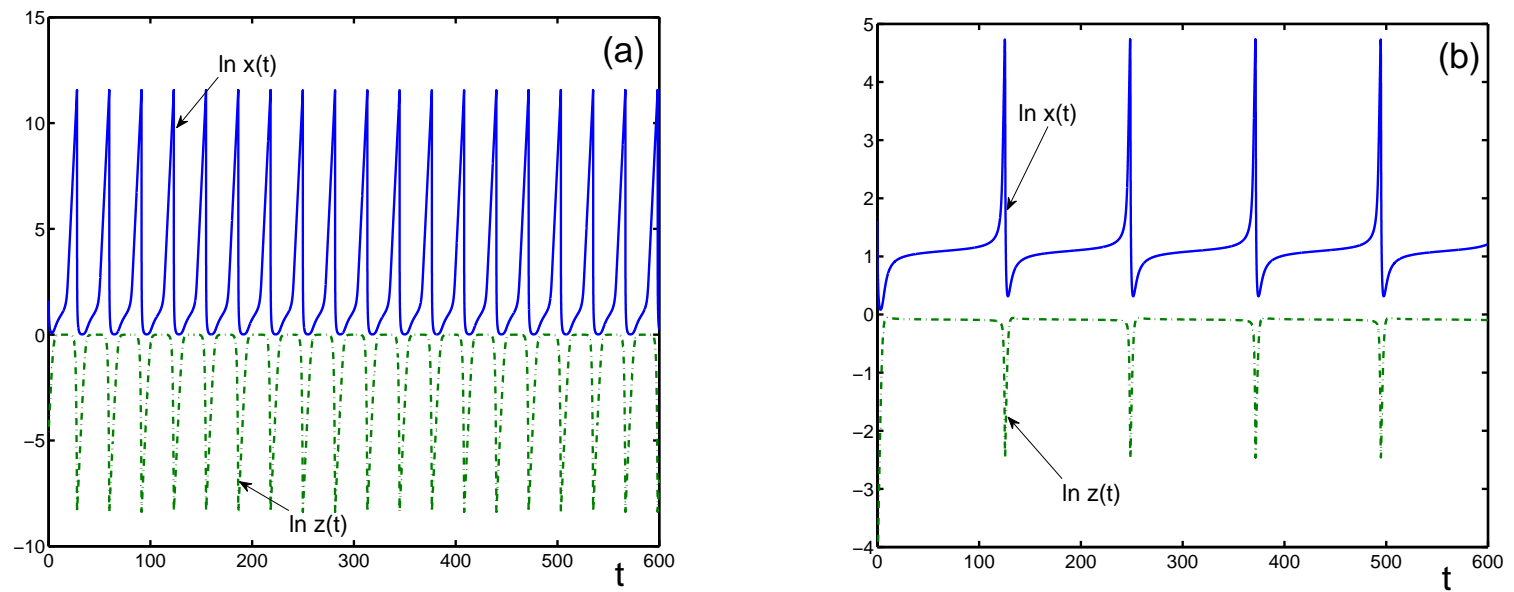

Fig. 17. Logarithmic behaviour of populations $x(t)$ (solid line) and $z(t)$ (dashed-dotted line) for $b=0.4$, and the initial conditions $\left\{x_{0}=5, z_{0}=0.01\right\}$, when $g_{c}(b) \approx-0.02942408$ and $g_{0}(b) \approx-0.05524965$. (a) Everlasting oscillations of populations for $g_{c}(b)<g=-0.0001<0$. (b) Everlasting oscillations of populations for $g_{0}(b)<g=-0.029<g_{c}(b)$. 

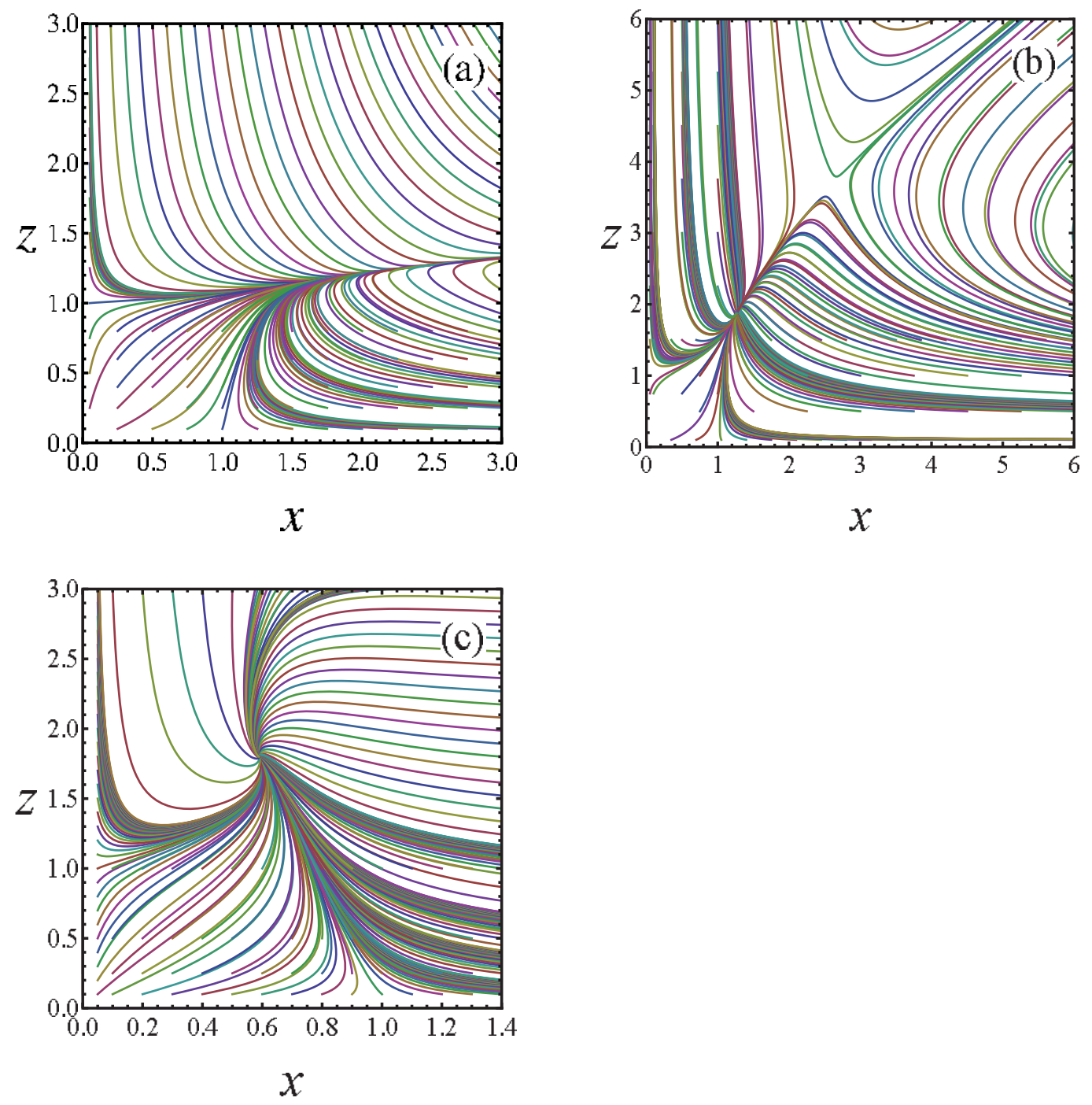

Fig. 18. Phase portrait for mixed symbiosis, with the symbiotic parameters $b$ and $g$ from qualitatively different regions: (a) Phase portrait for the symbiotic parameters, where there are no fixed points. The parameters are $b=0.3$ and $g=0.1>g_{c}(b)$. Here $g_{c}(b) \approx 0.0817$. (b) Phase portrait for the parameters $b=0.1$ and $g=0.5<g_{c}(b)$, with $g_{c}(b) \approx 0.673$, where there are two fixed points. The first fixed point $\left\{x_{1}^{*}=1.27133, z_{1}^{*}=1.8882\right\}$ is stable, with the Lyapunov exponents $\lambda_{1}=-1.28863$ and $\lambda_{2}=-0.471306$. The second fixed point $\left\{x_{2}^{*}=2.60324, z_{2}^{*}=3.67525\right\}$ is a saddle, with the Lyapunov exponents $\lambda_{1}=-1.73578$ and $\lambda_{2}=0.69254$. (c) Phase portrait for the parameters $b=-0.5$ and $g=1$, where there is a single fixed point $\left\{x^{*}=0.588548, z^{*}=1.80137\right\}$ that is a stable focus, with the characteristic exponents $\lambda_{1}=-1.26505-0.49167 i$ and $\left.\lambda_{2}=\lambda_{1}^{*}\right)$. 

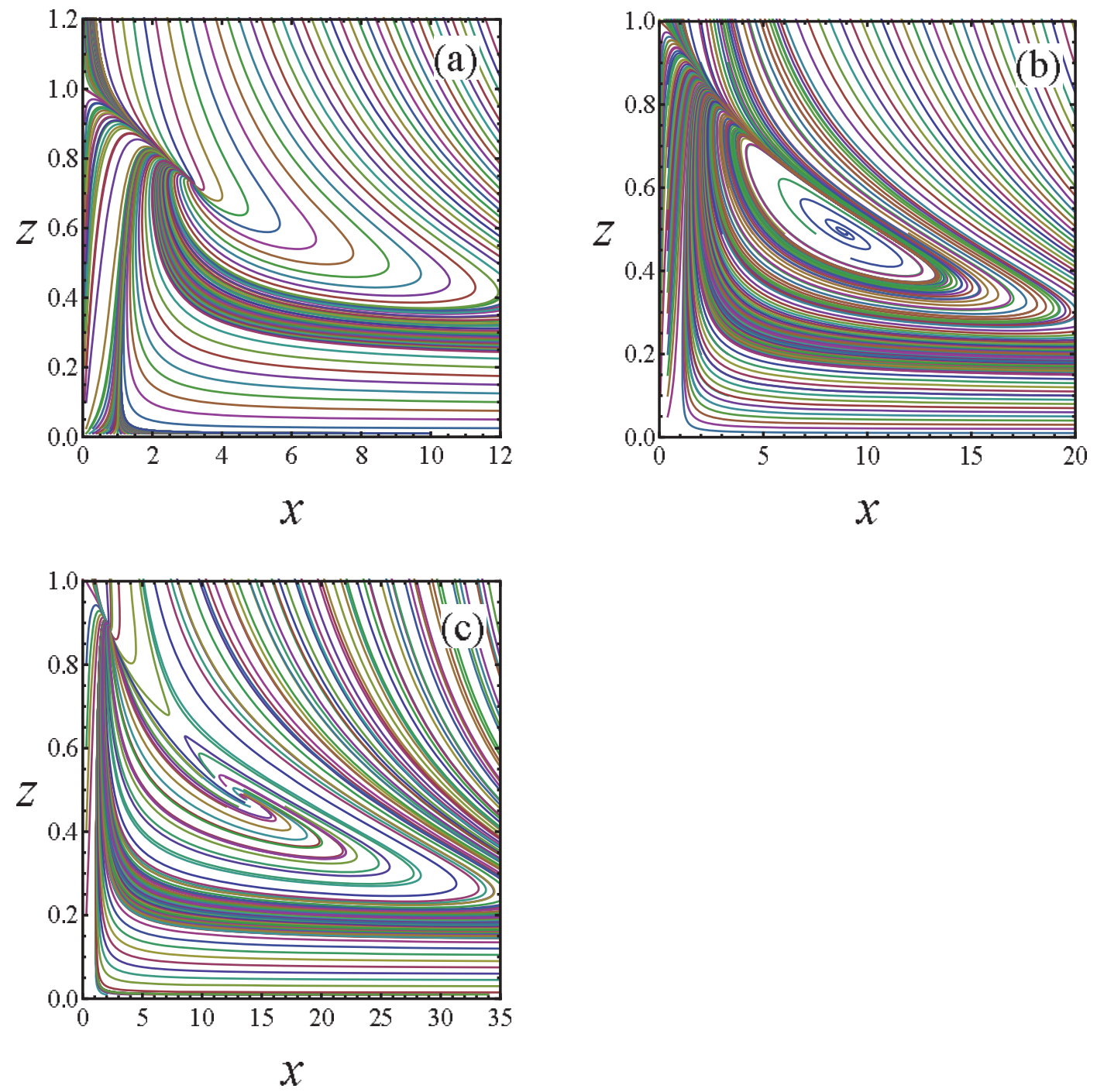

Fig. 19. Phase portrait for mixed symbiosis, with the symbiotic parameters $b>0$ and $g<0$ from qualitatively different regions. (a) Phase portrait for $b=0.5$ and $g=-0.1<g_{c}(b)<0$, with $g_{c}(b) \approx-0.083056$, in the region, where there is a single fixed point $\left\{x^{*}=3.1801, z^{*}=0.7276\right\}$ that is a stable focus, with the characteristic exponents $\lambda_{1}=-0.4215-0.1825 i$ and $\lambda_{2}=\lambda_{1}^{*}$. (b) Phase portrait for the same $b=0.5$, but with $g_{c}(b)<g=-0.083<0$. In this region, there exist a single fixed point $\left\{x^{*}=8.8369, z^{*}=0.49315\right\}$, which is an unstable focus, with the characteristic exponents $\lambda_{1}=0.08947-0.5945 i$, $\lambda_{2}=\lambda_{1}^{*}$, and a limit cycle. (c) Phase portrait for $b=0.4$ and $g=-0.054$, when $g_{c}(b) \approx-0.02943$ and $g_{0}(b) \approx-0.05524965$, so that $g_{0}(b)<g<g_{c}(b)<0$. The parameters are in the region of $b>0, g<0$, where three fixed points exist. One of them, $\left\{x_{1}^{*}=2.15176, z_{1}^{*}=0.890302\right\}$ is a stable node, with the Lyapunov exponents $\lambda_{1}=-0.85719$ and $\lambda_{2}=-0.37654$. The two other fixed points are unstable. The point $\left\{x_{2}^{*}=8.08875, z_{2}^{*}=0.646106\right\}$ is a saddle, with the Lyapunov exponents $\lambda_{1}=-0.37834$ and $\lambda_{2}=0.468814$. And $\left\{x_{3}^{*}=13.6002, z_{3}^{*}=0.479787\right\}$ is an unstable focus, with the characteristic exponents $\lambda_{1}=0.305044-0.462324 i$ and $\lambda_{2}=\lambda_{1}^{*}$. 

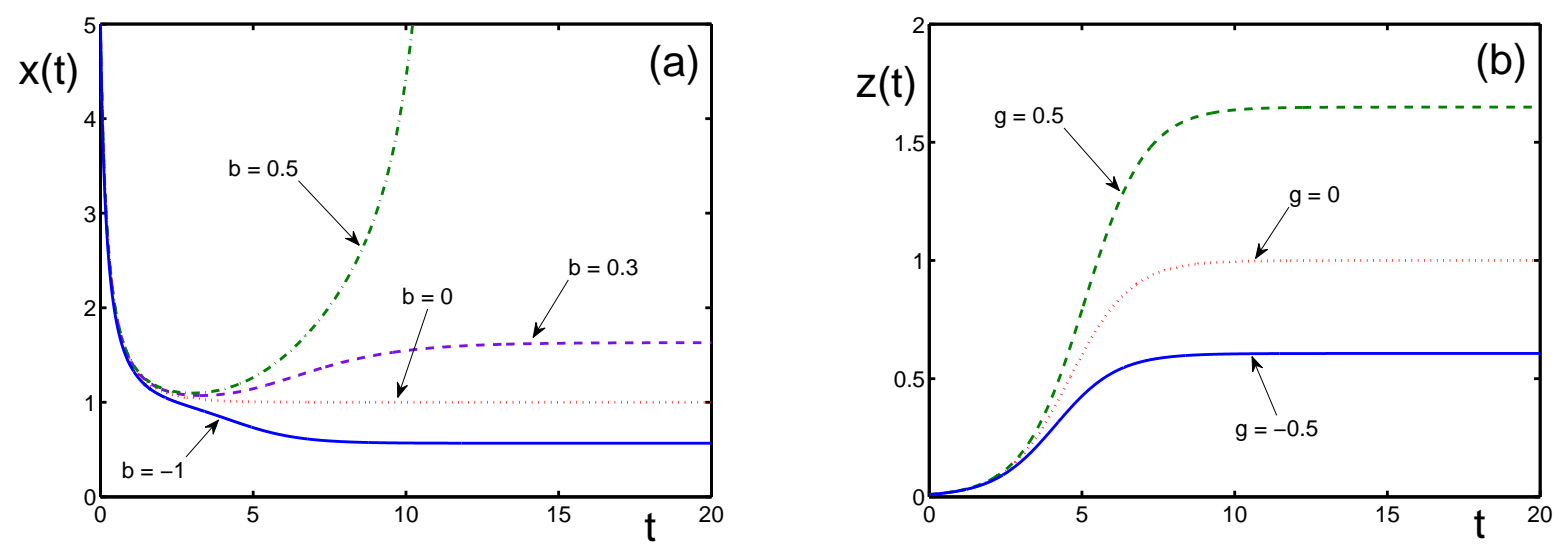

Fig. 20. Dynamics of populations under commensalism, characterized by the degenerate systems of equations (24), (26), and (27). Initial conditions are $\left\{x_{0}=5, z_{0}=0.01\right\}$. (a) Dynamics of the population $x(t)$ for different $b$ and $g=0$ : For $b=0$ (dotted line), $x(t) \rightarrow x^{*}=1$; for $b=0.5>1 / e$ (dashed-dotted line), $x(t) \rightarrow \infty$; for $b=0.3<1 / e\left(\right.$ dashed line), $x(t) \rightarrow x^{*}=1.63$; and for $b=-1$ (solid line), $x(t) \rightarrow x^{*}=0.567$, as $t \rightarrow \infty$. (b) Dynamics of the population $z(t)$ for different $g$ and $b=0$ : For $g=0$ (dotted line), $z(t) \rightarrow z^{*}=1$; for $g=0.5$ (dashed-dotted line), $z(t) \rightarrow z^{*}=e^{g}=1.65$; and for $g=-0.5$ (solid line), $z(t) \rightarrow z^{*}=0.607$, as $t \rightarrow \infty$. 